\title{
Effects of task probability on prioritized processing: Modulating the efficiency of parallel response selection
}

\author{
Jeff Miller ${ }^{1}$ (D) Jia Li Tang ${ }^{1}$ \\ Accepted: 7 September 2020 / Published online: 30 September 2020 \\ (C) The Psychonomic Society, Inc. 2020
}

\begin{abstract}
Four experiments investigated the extent to which a limited pool of resources can be shared between different tasks performed simultaneously when it is efficient to do so. The experiments used a prioritized processing paradigm, in which stimuli for both a primary task and a background task were presented in each trial. If the primary-task stimulus required a response in a trial, participants made only that response. If the primary-task stimulus did not require a response, participants responded to the background task. The main manipulation was the relative probability that a response would be required to the primary versus background task. In some blocks, the majority of trials required responses to the primary task (Experiments 1 and 2: 80\%; Experiments 3 and 4: 60\%), whereas in other blocks the majority required responses to the background task. Background-task responses were substantially faster in blocks where they were more likely to be required, consistent with the idea that more capacity was allocated to them in these blocks. Backward compatibility effects on primary-task responses and stimulus-onset asynchrony effects on background-task responses provided further evidence of greater capacity allocation to the background task when there was a higher probability of responding to it. The results support the view that two tasks can be processed in parallel, with resources divided between them, when it is efficient to do so.
\end{abstract}

Keywords Multitasking $\cdot$ Prioritized processing paradigm $\cdot$ Bottleneck models $\cdot$ Capacity models $\cdot$ Task probability

People are generally slower and less accurate when performing two or more cognitive tasks together than when performing each of the tasks individually, and this ubiquitous phenomenon is important for both practical and theoretical reasons (e.g., Pashler, 1994; Wickens, Goh, Helleberg, Horrey, \& Talleur, 2003). From a practical viewpoint, recent technological advances - particularly in communicationoffer greatly increased opportunities for multitasking (e.g., Gleick, 1999; Rosen, 2008). These same advances can also

These experiments were carried out by J.L.T. in partial fulfillment of the requirements for an MSc, under the supervision of J.M.

Electronic supplementary material The online version of this article (https://doi.org/10.3758/s13414-020-02143-7) contains supplementary material, which is available to authorized users.

Jeff Miller

miller@psy.otago.ac.nz

Jia Li Tang

jialitang1017@gmail.com

1 Department of Psychology, University of Otago, Dunedin 9054, New Zealand magnify the consequences of performance and thereby increase the costs resulting from any performance decrements due to multitasking. For example, with electronic communications, an erroneous message can be transmitted to thousands of recipients at once. At a theoretical level, understanding the causes of multitasking decrements is almost certain to be very revealing about the underlying architecture and processes of human cognition (e.g., Meyer \& Kieras, 1997a; Pashler, 1994). Despite many studies of these decrements, however, there remain fundamental disagreements about their causes.

One view is that performance is limited by an indivisible bottleneck process that must handle different tasks serially. In multitasking situations, performance is slowed because some tasks have to wait their turn for access to the bottleneck process, much as customers in a bank queue up to wait for a teller (Pashler, 1994). Based on a variety of empirical findings, this bottleneck process seems most clearly associated with the process of deciding how to respond, so the most common version of this model is called the central bottleneck or response selection bottleneck (RSB) model (e.g., Pashler, 1994; Schumacher et al., 1999). The RSB model has become the baseline model for the analysis of multitasking performance because it is conceptually simple, makes clear predictions 
about the effects of key experimental manipulations (e.g., Pashler, 1994), and is amenable to detailed quantitative analysis (e.g., Schwarz \& Ischebeck, 2001).

Alternatives to RSB models are more complex because they allow multiple responses to be selected in parallel (e.g., Navon, 1984). The most common of these are capacity models, in which multitasking performance worsens because a limited cognitive capacity must be divided among multiple tasks (e.g., Navon \& Miller, 2002; Tombu \& Jolicœur, 2003). When there are more tasks, each task is allocated less processing capacity, and that naturally reduces performance compared with single-task conditions in which each task is processed with full capacity. Thus, in contrast to serial bottleneck models, limited capacity models allow people to select multiple responses in parallel, albeit at a reduced rate.

Many studies contrasting bottleneck and capacity models have used a version of the classic psychological refractory period (PRP) paradigm (e.g., Welford, 1952). Stimuli ( $\mathrm{S}_{1}$ and $S_{2}$ ) for two separate tasks are presented successively, and participants are required to make separate responses $\left(\mathrm{R}_{1}\right.$ and $R_{2}$ ) in each of the two tasks, yielding separate response times $\left(\mathrm{RT}_{1}\right.$ and $\left.\mathrm{RT}_{2}\right)$ for the two tasks. A key feature of the paradigm is that the opportunity for multitasking can be tightly controlled by varying the stimulus-onset asynchrony (SOA) between the two stimuli, typically somewhere within the range of approximately $50-2,000 \mathrm{~ms}$.

Given the fundamental difference between serial bottleneck models and parallel capacity models, it might be surprising that there is still no consensus about when each model provides a better description of multitasking performance. Multitasking has been studied extensively from both perspectives (for a review see, e.g., Koch, Poljac, Müller, \& Kiesel, 2018), and there are certainly good arguments on each side.

Advocates of bottleneck models highlight various data patterns consistent with the idea that the responses of different tasks are selected serially (e.g., Pashler, 1992). Prominent among those patterns is the finding that second-task $\mathrm{RT}_{2}$ sometimes increases nearly millisecond for millisecond as SOA decreases (e.g., Telford, 1931) — a pattern known as the PRP effect. This is just the pattern that would be expected if second-task response selection simply has to wait for firsttask response selection to finish (i.e., wait for access to the bottleneck): As SOA is reduced, waiting time increases correspondingly.

Advocates of capacity models have at least two compelling replies to the arguments for bottleneck models. First, they argue that many of the data patterns cited as evidence of serial processing are actually quite compatible with models in which response selection is parallel to some degree (for reviews, see, e.g., Navon \& Miller, 2002; Tombu \& Jolicœur, 2003). Second, they emphasize that serial processing is always a strategic option within capacity models, so evidence of serial processing does not contradict them. That is, capacity models do allow people to adopt a strategy of selecting responses serially, simply by allocating $100 \%$ of capacity to each task in turn.

Capacity-model advocates argue further that if parallel response selection is actually possible, then the best way to observe it would be to set up conditions encouraging its use. There are good reasons to think that PRP paradigms do not do that, which may explain why processing appears to be serial in those paradigms. For example, with the standard instructions for PRP tasks, participants are explicitly told to give absolute priority to the first task and to respond to the second only after completing the first. Such a heavy emphasis on Task 1 could encourage participants to process the tasks serially even if parallel processing were possible (e.g., Meyer \& Kieras, 1997a; Schumacher et al., 2001). Advocates of bottleneck models have countered this argument by showing that eliminating the Task 1 emphasis from the PRP paradigm does not produce clear evidence of parallel processing (e.g., Levy \& Pashler, 2001, 2008; Levy, Pashler, \& Boer, 2006; Ruthruff, Pashler, \& Hazeltine, 2003b; Ruthruff, Pashler, \& Klaassen, 2001). Nonetheless, there are reasons to believe that in many PRP tasks serial processing is simply more efficient than parallel processing as a way to minimize overall mean RT (Hübner \& Lehle, 2007; Lehle \& Hübner, 2009; Miller, Ulrich, \& Rolke, 2009). This implies that participants might reasonably choose to process the tasks serially, even without strong incentives to do so.

Bottleneck-model advocates have also attempted to create task situations that would require parallel response selection, reasoning that these situations would offer the best opportunity for observing it if it were possible. For example, within a game-like dual-task situation, Ruthruff, Johnston, and Remington (2009) introduced severe response deadlines such that participants could only succeed in the game if they could select two independent responses in parallel just as quickly as they selected them in isolation. They argued that this would encourage parallel processing, because "if participants can overlap central operations without interference, then they should do so here" (p. 1371). Participants still showed substantial dual-task costs, however, so they were clearly not capable of selecting the two responses in parallel without interference. Whether that interference was caused by a structural bottleneck or by capacity limitations, however, remains an open question. Thus, setting up PRP paradigms that actually encourage parallel processing might be more difficult than it would first appear.

Often, advocates of capacity models point to evidence that there is parallel processing of the sort allowed by capacity models, but denied by bottleneck models. One source of evidence comes from backward compatibility effects (BCEs) on first-task response time, $\mathrm{RT}_{1}$ (for a recent overview, see, e.g., Janczyk, Renas, \& Durst, 2018). These are effects on $\mathrm{RT}_{1}$ of the compatibility between $\mathrm{R}_{1}$ and some $\mathrm{S}_{2}$ or $\mathrm{R}_{2}$ 
characteristics that should not be available while $R_{1}$ is being selected, at least according to bottleneck models. For example, Hommel (1998) asked participants to make left versus right key-press responses to red versus green stimuli as Task 1 and to give "left" versus "right" vocal responses to the letters $H$ versus $S$ as Task 2 . Task 1 responses were approximately 75ms faster when $R_{1}$ was compatible with the response word for Task 2 (e.g., a left key press for Task 1 and the response word "left" for Task 2) than when these were incompatible (e.g., a left key press for Task 1 and the response word "right" for Task 2). For that to happen, some information about the upcoming $\mathrm{R}_{2}$ must have been available before the end of the processes generating the earlier $\mathrm{R}_{1}$. Moreover, Hommel's result is far from unique, with various types of backward compatibility effects now having been reported in a wide variety of tasks (e.g., Ellenbogen \& Meiran, 2008; Fischer, Miller, \& Schubert, 2007; Hommel \& Eglau, 2002; Janczyk et al., 2018; Logan \& Delheimer, 2001; Logan \& Schulkind, 2000; Miller, 2006, 2017; Miller \& Alderton, 2006; Thomson, Watter, \& Finkelshtein, 2010; Watter \& Logan, 2006).

It is easy to see how BCEs could arise within the PRP paradigm if the two responses were selected in parallel, since that would make it easy for information about $\mathrm{R}_{2}$ to become available in time to influence $R_{1}$. A more difficult question is whether these effects might also be reconciled with the serial bottleneck models that have otherwise been rather successful in accounting for PRP task phenomena. In his original report of the BCE, Hommel (1998) suggested a bottleneck account in which responses were activated for both tasks in parallel, even though they were only selected for one task at a time due to the bottleneck limitation. On this account, Task 1 responses would be speeded by congruent activations in compatible trials and slowed by incongruent activations in incompatible trials, producing the $\mathrm{BCE}$. Yet the serial character of the response selection process would be preserved, in keeping with RSB models, because the BCE would arise from some automatic process - not response selection. More generally, the BCE can be reconciled with serial models if the early $R_{2}$ information arises from any type of automatic processing that does not require the bottleneck (e.g., Miller \& Alderton, 2006). Recent results from three-task paradigms suggest that the BCE arises because of an effect on capacity-limited Task 1 processes, but these results are compatible with the view that the effect is produced by automatic, capacity-unlimited processing of $\mathrm{S}_{2}$, so they do not rule out bottleneck models (Janczyk et al., 2018).

A relatively new experimental approach that attempts to document flexible capacity allocation more directly has been to examine the effects of various manipulations designed to increase parallel processing (for a review, see Fischer \& Plessow, 2015), and many of these have used the BCE as an indicator of parallel processing. For example, Lehle and colleagues simply instructed participants to perform the two tasks serially in some blocks of trials and to perform them in parallel in other blocks (Lehle \& Hübner, 2009; Lehle, Steinhauser, \& Hübner, 2009). Consistent with capacity models, they found larger BCEs in blocks with parallel instructions. They also found, however, that responses were generally slower and less accurate with parallel instructions, suggesting that parallel processing was less efficient overall. This raises the alternative possibility that performance actually is limited by a serial bottleneck, in which case participants would have been forced to adopt artificial and somewhat inefficient strategies in their attempts to comply with impossible instructions - that is, instructions to process in parallel. For instance, they might sometimes have grouped responses (e.g., Borger, 1963; Pashler \& Johnston, 1989; Ulrich \& Miller, 2008) or performed the tasks in reversed order (e.g., Leonhard, Ruiz Fernández, Ulrich, \& Miller, 2011). They might adopt such strategies in an attempt to mimic parallel processing, even if the choice to process in parallel is not under voluntary control.

Other experiments have used a variety of manipulations intended to influence the balance between serial and parallel processing. It has been suggested that the degree of parallelism can be modulated by stress (Beste, Yildiz, Meissner, \& Wolf, 2013; Plessow, Schade, Kirschbaum, \& Fischer, 2012), by the presentation of high-frequency binaural beats (Hommel, Sellaro, Fischer, Borg, \& Colzato, 2016), or by using different kinds of problem-solving tasks to induce divergent versus convergent thinking styles prior to the start of the dual-task experiment (Fischer \& Hommel, 2012). In general, the BCE is found to increase in the conditions where more parallel processing is expected, as predicted by capacity models. In keeping with bottleneck models, however, it still seems possible that the observed BCE is driven by automatic, capacity-free processes and that changes in it are driven by other systemic effects of these manipulations (e.g., changes in reactivity to response activation) rather than by an increase in parallel response selection.

Assuming that people try to use their limited cognitive resources optimally (e.g., Lieder \& Griffiths, 2020), they should be especially likely to select responses in parallel when parallel processing — if it is possible at all —would be more efficient than serial processing. For example, there are good reasons to predict that parallel processing would tend to be more efficient when short SOAs are especially common (Miller et al., 2009; see also Leonhard et al., 2011). Consistent with that prediction, the strong PRP effect predicted by serial models (i.e., sharp increase in $\mathrm{RT}_{2}$ at short SOAs) is weakened when short SOAs are more common than long ones (Mattes et al., 2020; Miller et al., 2009; Yildiz \& Beste, 2015), thus suggesting that processing does become more parallel when that would be more efficient.

Researchers have also manipulated the frequency of backward-incompatible trials to alter the relative efficiency of serial and parallel response selection (e.g., Fischer, 
Gottschalk, \& Dreisbach, 2014). Intuitively, when backwardcompatible trials are frequent, parallel response selection would be relatively efficient because Task 2 response activations would usually facilitate $\mathrm{R}_{1}$. When backward-compatible trials are infrequent, in contrast, Task 2 activations would usually inhibit the correct $\mathrm{R}_{1}$. In that case, serial response selection would be more efficient, because it would minimize this inhibition. As expected from this analysis, Fischer et al. (2014) found that the BCE is larger for stimuli appearing in locations where backward-compatible trials are more frequent. Similarly, even when backward-compatible and backward-incompatible trials are equally frequent in a given location, the BCE is larger in a trial following a backwardcompatible trial than following a backward-incompatible trial (e.g., Janczyk, 2013, 2016; Scherbaum, Gottschalk, Dshemuchadse, \& Fischer, 2015). Such findings are certainly consistent with models in which the degree of parallel response selection can be adjusted to suit the experimental context. They can still be reconciled with serial response selection, however, under the assumption that the influence of automatic processes giving rise to the $\mathrm{BCE}$ is sensitive to the experimental context - an assumption that is supported by ample findings of congruency proportion and sequence effects in Stroop, Simon, and Eriksen tasks (e.g., Gratton, Coles, \& Donchin, 1992; Logan \& Zbrodoff, 1979; Stürmer, Leuthold, Soetens, Schröter, \& Sommer, 2002).

\section{Present experiments}

These experiments focused on the question of whether the extent of parallel response selection can be directly influenced by the relative efficiency of serial versus parallel task processing (cf. Miller et al., 2009). That is, we sought to produce conditions under which it would be particularly efficient to divide resources between two tasks and process them in parallel, assuming that this would produce clear evidence of capacity sharing and parallel response selection, if that is indeed possible. Compared with previous studies, the procedures of the present experiments were novel with respect to the multitasking paradigm used, the task probabilities, and the indicators of parallel processing. These differences are discussed in the next three sections.

Prioritized processing paradigm These experiments used the prioritized processing (PP) paradigm (Miller \& Durst, 2014, 2015) instead of the PRP paradigm. The PP paradigm involves two tasks - a primary task and a background taskand it is similar to the standard PRP paradigm in that the task order is fixed. Specifically, the participant must always process the primary task first, proceeding to the background task only after the primary task is finished. The PP paradigm differs from the PRP paradigm in that it requires only one motor response per trial- either to the primary task or to the background task, but never to both. Thus, this paradigm also has similarities to other dual-task paradigms in which participants must be prepared to perform either of two tasks in each trial, but make only one response (e.g., task-switching, Koch et al., 2018; stop-change, Logan \& Burkell, 1986). One advantage of this single-response procedure is that eliminates betweentask motor interference that may exaggerate dependencies between the RTs of the two tasks (e.g., Borger, 1963; Pashler \& Johnston, 1989; Ulrich \& Miller, 2008). A more important advantage is that it provides a robust manipulation of task emphasis that can be used to modulate the relative importance of the tasks — namely, task probability —as is discussed in the next section.

In each of the current experiments, two stimuli-one for each task - were presented in each trial, separated by an SOA of 50 or $200 \mathrm{~ms}$, with the primary-task stimulus, $\mathrm{S}_{p}$, always presented first. Participants were instructed that the highest priority was to respond to the primary-task stimulus. This could be a stimulus assigned to either a left-hand key-press response, a right-hand key-press response, or a no-go response. When presented with either of the stimuli assigned to key-press responses (i.e., primary-task go stimuli), the participant simply had to make the indicated response. After that response, $\mathrm{R}_{p}$, whose latency we denote as $\mathrm{RT}_{p}$, the trial was finished, with no response required to the background-task stimulus, $\mathrm{S}_{b}$. When a primary-task no-go stimulus was presented, however, participants were required to make a lefthand or right-hand key-press response based on the identity of the background-task stimulus, $\mathrm{S}_{b}$, thus making a background-task response, $\mathrm{R}_{b}$, instead of a primary-task response. The RTs in these trials, $\mathrm{RT}_{b}$, were measured relative to the onset of the background-task stimulus.

For example, in Experiment 1, the primary-task stimulus, $\mathrm{S}_{p}$, was a green, red, or blue outline square, and the background task stimulus, $\mathrm{S}_{b}$, was a white two-digit number presented inside that square. One color was assigned to the lefthand response, one to the right-hand response, and one to the no-go response, randomizing color assignments across participants. When the square appeared in either of the colors assigned to key-press responses, participants simply had to press the appropriate key, thus making a primary-task response, $\mathrm{R}_{p}$, and producing a primary-task $\mathrm{RT}_{p}$. In such trials the background task number stimulus could be ignored.

When the square appeared in the no-go color, however, participants had to respond on the basis of the number, responding with the left hand if it was less than 45 and with the right hand if it was greater than 45 (cf. Hinrichs, Yurko, \& $\mathrm{Hu}, 1981$; Sigman \& Dehaene, 2005). Thus, in these trials, participants made a background-task response, $\mathrm{R}_{b}$, and produced a background-task $\mathrm{RT}_{b}$.

Because it requires only one response per trial, the PP paradigm avoids problems of response grouping (e.g., Borger, 
1963; Ulrich \& Miller, 2008), motor interference (e.g., Bratzke, Rolke, \& Ulrich, 2009), and action-effect monitoring (e.g., Kunde, Wirth, \& Janczyk, 2018) that can complicate the interpretation of results in PRP paradigms. Most importantly for the present purposes, task priority can be manipulated more effectively in the PP paradigm than in the PRP paradigm because the requirement to make only a single response means that the two tasks are mutually exclusive and strictly trade off against one another. The present experiments leveraged this aspect of the PP paradigm by varying task probability to manipulate task emphasis, as is discussed next.

Task probability manipulation The critical experimental manipulation in these studies involved the relative probability that a response would be required in each of the two tasks. In High-Primary blocks, the primary-task stimuli that were presented required responses in most trials. In these blocks, it would be most efficient to devote full resources to processing the primary task, because that would minimize the time needed for these frequent responses (cf. Logan \& Gordon, 2001; Miller et al., 2009). Thus, serial processing would be expected in these blocks, whether it was obligatory (bottleneck models) or optional (capacity models).

In the High-Background blocks of main interest, however, relatively few primary-task stimuli required responses (i.e., most were no-go primary-task stimuli). As a consequence, most trials required responses to the background task in these blocks. In these blocks, it would seem most efficient to select responses in parallel, with considerable capacity allocated to the background task, since most responses would be made to that task. Thus, our main questions concerned the extent to which indications of parallel processing would emerge in the High-Background blocks, consistent with the idea that efficiency is an important determinant of capacity allocation and serial versus parallel response selection in multitasking.

Indicators of parallel processing Three distinct data patterns, each of which violates a prediction of serial bottleneck models, could potentially reveal more extensive parallel processing in the High-Background blocks than in the High-Primary blocks. Figure 1 illustrates these predictions by showing the stage sequences predicted by the bottleneck model for the PP paradigm (cf. Miller \& Durst, 2015). The effects of task probability are represented within the model by changes in the processing durations of the individual stages, with each task's stages taking less time when it is the high-probability task. Without loss of generality, it is convenient to regard the stage durations for a given task as being at baseline when that task has high probability and to increase when the task has low probability. Thus, $\mathrm{A}_{p}, \mathrm{~B}_{p}$, and $\mathrm{C}_{p}$ represent the durations of the primary-task perception, response selection, and motor execution stages in the High-Primary blocks, and these durations increase to $\mathrm{A}_{p}{ }_{p}=$ $\mathrm{A}_{p}+\Delta_{\mathrm{Ap}}, \mathrm{B}^{*}{ }_{p}=\mathrm{B}_{p}+\Delta_{\mathrm{Bp}}$, and $\mathrm{C}_{p}^{*}=\mathrm{C}_{p}+\Delta_{\mathrm{Cp}}$ in the High-
Background blocks. Analogously, $\mathrm{A}_{b}, \mathrm{~B}_{b}$, and $\mathrm{C}_{b}$ represent the durations of the background task stages in the HighBackground blocks, and these are assumed to increase to $\mathrm{A}_{b}^{*}=\mathrm{A}_{b}+\Delta_{\mathrm{Ab}}, \mathrm{B}^{*}{ }_{b}=\mathrm{B}_{b}+\Delta_{\mathrm{Bb}}$, and $\mathrm{C}^{*}{ }_{b}=\mathrm{C}_{b}+\Delta_{\mathrm{Cb}}$ in the High-Primary blocks. We assume that all of the $\Delta$ s are positive or zero, which means that no stage operates faster when task probability is low, although one or two could operate at the same speed.

A first potential indicator of parallel response selection in the High-Background blocks is a flattening of the slope relating $\mathrm{RT}_{b}$ to SOA (e.g., Mattes et al., 2020; Miller et al., 2009; Yildiz \& Beste, 2015). As discussed previously, with serial response selection, Stage $\mathrm{B}_{b}$ must wait for the completion of Stage $\mathrm{B}_{p}$, so the RSB model always predicts a -1 slope at short SOAs. Furthermore, this model predicts that the slope should be the same in both High-Primary and High-Background blocks, because response selection must be serial in both blocks. This is most clearly reflected in the equations for $\mathrm{RT}_{b}$ in the two blocks, both of which include the SOA term with a -1 coefficient (see Fig. $1 \mathrm{~b}$ and d). With the parallel processing allowed by capacity models, however, Stage $\mathrm{B}_{b}$ can start before Stage $\mathrm{B}_{p}$ is finished. At short SOAs, some Stage $\mathrm{B}_{b}$ work could then be carried out while Stage $\mathrm{B}_{p}$ was still underway, so $\mathrm{RT}_{b}$ could be less affected by waiting. In short, if there is increased parallel response selection in High-Background blocks, then the slope relating $\mathrm{RT}_{b}$ to SOA might well be shallower in these blocks than in the High-Primary blocks. ${ }^{1}$

A second possible indicator of parallel response selection in the High-Background blocks would be an increase in the BCE in these blocks relative to the High-Primary blocks. As discussed earlier, the BCE is a plausible indicator of parallel processing (e.g., Fischer et al., 2014; Fischer \& Hommel, 2012; Plessow et al., 2012; Zwosta, Hommel, Goschke, \& Fischer, 2013; for a review, see Fischer \& Plessow, 2015), although it is not clear whether the parallel second-task process responsible for the $\mathrm{BCE}$ is response selection per se or some automatic process. If the $\mathrm{BCE}$ does arise because of parallel response selection in accordance with capacity model accounts, then it should certainly be larger when more resources are allocated to background-task response selection in the High-Background blocks. With more resources, the background-task response would be selected sooner and thus have more time to influence primary-task response selection. This would tend to increase facilitation in backwardcompatible trials and inhibition in backward-incompatible

\footnotetext{
${ }^{1}$ The Appendix examines the possibility that participants might process in accordance with the RSB model in the High-Primary blocks, but might reverse the order of Stage B processing in the High-Background blocks - that is, they might process Stage $\mathrm{B}_{b}$ before Stage $\mathrm{B}_{p}$ in at least some trials-which might also produce a shallower slope in the High-Background blocks. Readers interested in a detailed analysis of this possibility should consult the Appendix, but the bottom line is that the data from the present experiments provide strong evidence that this did not happen often enough to explain any of the key results.
} 




C HiBac, $\mathbf{R T}_{\mathrm{p}}$

\begin{tabular}{|l|l|l|}
\hline $\mathrm{A}_{\mathrm{p}}^{*}$ & $\mathrm{~B}_{\mathrm{p}}^{*}$ & $\mathrm{C}_{\mathrm{p}}^{*}$ \\
\hline
\end{tabular}

$\operatorname{soA} A_{b}$

$$
\mathrm{RT}_{\mathrm{p}, \mathrm{HiBac}}=\mathrm{A}_{\mathrm{p}}^{*}+\mathrm{B}_{\mathrm{p}}^{*}+\mathrm{C}_{\mathrm{p}}^{*}
$$

Fig. 1 Illustration of hypothetical stage processing sequences leading to task probability effects (High-Primary [HiPri] versus High-Background [HiBac]) on $\mathrm{RT}_{p}$ and $\mathrm{RT}_{b}$ within the bottleneck model, assuming that the stage times for each task decrease when that task is highly probable. a-b Sequences producing primary-task $\mathrm{RT}_{p}$ and background-task $\mathrm{RT}_{b}$, respectively, when there is a high probability of responding to the primary task (HiPri). Within each panel, the top row shows primarytask processing stages, the middle row shows background-task processing stages, and the equation at the bottom shows how the total

trials - that is, to increase the BCE. Alternatively, it is also possible that task probability would modulate the effects of automatic second-task processes (cf. Giammarco, Thomson, \& Watter, 2016; Thomson, Danis, \& Watter, 2015), so an increased BCE in the High-Background blocks would not necessarily contradict RSB models that explain the BCE with such processes. Nonetheless, since the capacity model makes the clear prediction that the BCE should increase in the HighBackground blocks, we naturally checked this prediction in the current experiments.

The third and most novel indicator of parallel response selection in the current experiments is the effect of task probability on $\mathrm{RT}_{p}$ and $\mathrm{RT}_{b}$. As is explained next, under plausible conditions, models with serial response selection predict that task probability should have a much larger effect on $\mathrm{RT}_{p}$ than on $\mathrm{RT}_{b}$, whereas models with parallel response selection need not make this prediction. Hence, finding approximately equal effects of probability on the RTs of the two tasks would also tend to support capacity models.

The serial bottleneck models' prediction can be inferred from Fig. 1. The effect of task probability on $\mathrm{RT}_{p}$ is illustrated by the comparison between Fig. 1a and c. In High-Primary blocks, $\mathrm{RT}_{p, H i P r i}$ is the sum of the shorter stage times $\mathrm{A}_{p}+\mathrm{B}_{p}$ $+\mathrm{C}_{p}$ (see Fig. 1a), whereas in High-Background blocks, $\mathrm{RT}_{p, H i B a c}$ is the sum of the lengthened stages times $\mathrm{A}_{p}^{*}+$ $\mathrm{B}^{*}{ }_{p}+\mathrm{C}^{*}{ }_{p}$ (see Fig. 1c). Thus, the overall effect of task



\section{d HiBac, $\mathbf{R T}_{\mathbf{b}}$}



$\mathrm{RT}_{p}$ or $\mathrm{RT}_{b}$ is produced. c-d Sequences producing $\mathrm{RT}_{p}$ and $\mathrm{RT}_{b}$, respectively, when there is a high probability of responding to the background task (HiBac). Primary-task stage times are assumed to be shorter when there is a high probability of responding to the primary task (HiPri: $\mathrm{A}_{p}, \mathrm{~B}_{p}$, and $\mathrm{C}_{p}$ ) than when there is a high probability of responding to the background task ( $\mathrm{HiBac}: \mathrm{A}_{p}^{*}, \mathrm{~B}_{p}{ }_{p}$, and $\mathrm{C}_{p}^{*}$ ). The reverse is true for the background-task stage times; these are longer in the HiPri condition $\left(\mathrm{A}_{b}^{*}, \mathrm{~B}_{b}{ }_{b}\right.$, and $\left.\mathrm{C}_{b}{ }_{b}\right)$ than in the HiBac condition $\left(\mathrm{A}_{b}, \mathrm{~B}_{b}\right.$, and $\left.\mathrm{C}_{b}\right)$

probability on $\mathrm{RT}_{p}$ is the total of all low-probability slowing across the three primary-task stages, namely,

$\mathrm{RT}_{p, H i B a c}-\mathrm{RT}_{p, H i P r i}=\Delta_{\mathrm{Ap}}+\Delta_{\mathrm{Bp}}+\Delta_{\mathrm{Cp}}$.

The predicted task probability effect is quite different for $\mathrm{RT}_{b}$. When the SOA is short enough that Stage $\mathrm{B}_{b}$ must wait until Stage $\mathrm{B}_{p}$ finishes, as depicted in Fig. $1 \mathrm{~b}$ and d, $\mathrm{RT}_{b}$ is affected by the durations of the primary-task stages $\mathrm{A}_{p}$ and $\mathrm{B}_{p}$, because these durations influence the wait for the bottleneck. As a consequence, $\mathrm{RT}_{b}$ should be the sum of two shorter stage times and two longer ones both in High-Primary blocks (i.e., $\mathrm{RT}_{b, H i P r i}=\mathrm{A}_{p}+\mathrm{B}_{p}+\mathrm{B}_{b}^{*}+\mathrm{C}_{b}^{*}$ ) and in High-Background blocks (i.e., $\mathrm{RT}_{b, H i B a c}=\mathrm{A}_{p}^{*}+\mathrm{B}_{p}^{*}+\mathrm{B}_{b}+\mathrm{C}_{b}$ ). The task probability effect (High-Primary minus High-Background) is thus

$\mathrm{RT}_{b, H i P r i}-\mathrm{RT}_{b, H i B a c}=\Delta_{\mathrm{Bb}}+\Delta_{\mathrm{Cb}}-\Delta_{\mathrm{Ap}}-\Delta_{\mathrm{Bp}}$.

It seems plausible that the effect on $\mathrm{RT}_{p}$ shown in Eq. 1 should be larger than the effect on $\mathrm{RT}_{b}$ shown in Eq. 2, at least assuming that the processing stages of both tasks are slowed comparably by low task probability. In this case, then, bottleneck models predict that $\mathrm{RT}_{p}$ should change much more than $\mathrm{RT}_{b}$ between the High-Primary and High-Background blocks - at least when SOA is short-and a violation of this prediction would thus support the idea of parallel processing.

In contrast, parallel capacity models are not constrained to predict a larger effect of task probability on $\mathrm{RT}_{p}$ than on $\mathrm{RT}_{b}$. 
Since these models allow the response selection stages of the two tasks to be carried out in parallel, the durations of $\mathrm{A}_{p}$ and $\mathrm{B}_{p}$ do not simply add into $\mathrm{RT}_{b}$ as they do in RSB models, and the slowing of these stages in the High-Background conditions does not directly counteract the speeding of background task stages, as it does in Eq. 2.

\section{Experiment 1}

This experiment was an initial attempt to vary the probability of responding to the primary versus background task within the PP paradigm in order to check for indications that the tasks are processed in parallel when it is efficient to do so. In each trial, a colored outline square was presented with a two-digit number inside it, and participants were required to respond with a left or right index finger key press. The primary task was choice/no-go color discrimination, with one square color assigned to the left-hand response, one to the right-hand response, and one to the no-go response. The background task was number magnitude judgment, with numbers less than 45 assigned to the left-hand response and numbers greater than 45 assigned to the right-hand response. Participants were instructed that they should respond only to the primary color task in trials where the square appeared in the color assigned to the left or right response, ignoring the number in those trials. They were told to respond to the background number task only when the square appeared in the no-go color.

The probability of responding to the primary versus background task was varied between two types of trial blocks. In the High-Primary blocks, $80 \%$ of trials required responses to the primary color task and only $20 \%$ required responses to the background number task. In the High-Background blocks, these probabilities were reversed.

Three further effects were assessed via within-block comparisons. Two of these, discussed already, were the effects of SOA and backward compatibility. We used only the relatively short SOAs of 50 versus $200 \mathrm{~ms}$, because the RSB model only predicts a-1 slope of $\mathrm{RT}_{b}$ versus SOA when SOA is short and because BCEs tend to be larger when SOA is short. Based on previous results, we expected to find a clear effect of SOA on $\mathrm{RT}_{b}$ over this range, but little if any change in the BCE (e.g., Miller \& Durst, 2014, 2015). The third effect was the numeric distance effect (e.g., Hinrichs et al., 1981). Specifically, for trials requiring a response to the background number task, the number could be relatively near the decision boundary of 45 (e.g., 51) or it could be relatively far from that boundary (e.g., 71). Previous studies with similar tasks suggest that there should be a numerical distance effect such that number-task responses would be faster for numbers far from the boundary than for numbers near the boundary (e.g., Hinrichs et al., 1981; Reike \& Schwarz, 2016). This effect is not directly useful in discriminating between bottleneck and capacity models, but it is inherent in the numerical discrimination task and its relation to task probability provides some new evidence about numerical discrimination processes, as will be considered in the General Discussion.

\section{Method}

Participants Lacking information about the error terms for the theoretically critical interactions (i.e., Backward Compatibility $\times$ Task Probability and SOA $\times$ Task Probability), we planned to obtain a sample of approximately 60 participants, corresponding to the larger of the studies reported by Miller and Durst (2014), although we expected a small deviation from that target sample size based on practical difficulties involving participant scheduling and attendance. If the error terms for these interactions matched the error terms for the backward compatibility and SOA effects found in the earlier studies, this sample size would produce more than $85 \%$ power to detect a change in either effect size of $25 \mathrm{~ms}$.

The reported data were obtained from 62 volunteer participants (ages 18-23 years, $M=19.5$ years, 54 females) recruited at the University of Otago, who participated in partial fulfillment of an introductory psychology class requirement. All participants had normal or corrected-to-normal vision and were right-handed as measured by the Edinburgh Handedness Inventory (Oldfield, 1971), with a mean handedness score of $M=72.6$. Each participant attended one experimental session which lasted approximately $45 \mathrm{~min}$. Based on previous studies with the PP paradigm, we planned to exclude any participants whose overall accuracy level fell below $80 \%$, but all participants achieved this cutoff.

Apparatus, stimuli, and tasks Stimuli were presented and behavioral responses were recorded with an IBM-PC compatible computer running under the Ubuntu operating system. The experiment was controlled with a MATLAB program using the Psychophysics Toolbox extensions (Brainard, 1997; Kleiner et al., 2007; Pelli, 1997). All stimuli were bright figures appearing on an otherwise black computer monitor, centered at fixation and viewed from a distance of approximately $60 \mathrm{~cm}$. Responses were made with the left and right index fingers by pressing the $Z$ and / (slash) keys on a standard English computer keyboard.

Participants were required to perform a primary color discrimination task and a background number comparison task in a standard PP design. The primary-task stimuli were colored outline squares, $2.5^{\circ}$ on a side, made from green, red, or blue lines that were $0.2^{\circ}$ in thickness. The three colors were randomly assigned to the left, right, and no-go responses separately for each participant. The background task stimuli were two-digit numbers 21-69, excluding 45, that appeared in the centers of the squares in a white 28-point Tunga font. All participants were instructed to respond with the left index 
finger if the number was less than 45 and with the right index finger if it was greater than 45 .

Procedure Each participant was tested in eight blocks of trials of the types shown in Table 1, with the first four blocks in the High-Primary condition and the last four in the HighBackground condition, or the reverse, counterbalanced across participants. The first block in each probability condition was a practice block with 40 trials, and this was followed by three experimental blocks with 80 trials each. In the High-Primary experimental blocks, each of the trial types requiring a response to the primary task was tested eight times, and each of the trial types requiring a response to the background task was tested four times, so a primary-task response was required in $80 \%$ of trials. In the High-Background experimental blocks, each of the trial types requiring a response to the primary task was tested twice, and each of the trial types requiring a response to the background task was tested 16 times, so a background-task response was required in $80 \%$ of trials. Each type of trial was tested half as many times in the HighPrimary and High-Background practice blocks. Trial order was randomized separately for each block.

Each block started with an instructional screen telling participants to respond with the left or right hand to squares of two colors (e.g., left for red, right for green). They were told

Table 1 Examples of the different possible trial types in Experiment 1

\begin{tabular}{|c|c|c|c|c|}
\hline \multicolumn{2}{|l|}{ Stimuli } & \multirow[b]{2}{*}{$\begin{array}{l}\text { Correct } \\
\text { response }\end{array}$} & \multicolumn{2}{|c|}{ Within-block effects } \\
\hline $\begin{array}{l}\text { Primary } \\
\text { task }\end{array}$ & $\begin{array}{l}\text { Background } \\
\text { task }\end{array}$ & & $\begin{array}{l}\text { Backward } \\
\text { compatibility }\end{array}$ & $\begin{array}{l}\text { Numerical } \\
\text { distance }\end{array}$ \\
\hline \multicolumn{5}{|c|}{ Primary-task responses } \\
\hline Green & $21-32$ & Left & Compatible & Far \\
\hline Green & $33-44$ & Left & Compatible & Near \\
\hline Green & $46-57$ & Left & Incompatible & Near \\
\hline Green & $58-69$ & Left & Incompatible & Far \\
\hline Red & $21-32$ & Right & Incompatible & Far \\
\hline Red & $33-44$ & Right & Incompatible & Near \\
\hline Red & $46-57$ & Right & Compatible & Near \\
\hline Red & $58-69$ & Right & Compatible & Far \\
\hline \multicolumn{5}{|c|}{ Background-task responses } \\
\hline Blue & $21-32$ & Left & n.a. & Far \\
\hline Blue & $33-44$ & Left & n.a. & Near \\
\hline Blue & $46-57$ & Right & n.a. & Near \\
\hline Blue & $58-69$ & Right & n.a. & Far \\
\hline
\end{tabular}

Note. Examples of the different conditions for a participant instructed to respond with the left hand if a green square was presented, to respond with the right hand if a red square was presented, and to respond by classifying the number as smaller than 45 (left hand) or larger than 45 (right hand) if a blue square was presented. For each of these types of trials, half of the trials in each block were tested at a stimulus-onset asynchrony (SOA) of $50 \mathrm{~ms}$, and half at an SOA of $200 \mathrm{~ms}$ that if the square was the other color (e.g., blue), they should respond based on the two-digit number.

Each trial began with the presentation of a fixation plus sign at the center of the computer screen for $500 \mathrm{~ms}$. Then the square and number were presented, in that order, separated by a randomly varying SOA of either $50 \mathrm{~ms}$ or $200 \mathrm{~ms}$. These stimuli remained on screen until the response was made or until $3 \mathrm{~s}$ had elapsed, whichever came first. Accuracy feedback was displayed for $1 \mathrm{~s}$ following a correct response and for $3 \mathrm{~s}$ following an error, with this difference in feedback duration used to encourage accurate responding. The plus sign reappeared to begin the next trial approximately $1 \mathrm{~s}$ after the offset of the accuracy feedback.

Data analysis In the initial practice block of each task probability condition (i.e., High-Primary versus High-Background), participants would have had to learn the prevailing task probabilities and adapt their performance accordingly, so this block was omitted from the final analyses. Error trials were excluded from the analyses of RTs, and obtained RT distributions were visually inspected to identify cutoffs for RT outliers. The mean correct response times (RT) and percentages of correct responses (PC) for the remaining trials were computed for each participant in each condition, and these were submitted to analyses of variance (ANOVAs).

Two ANOVAs focused on the effects of task probability on $\mathrm{RT}_{p}$ versus $\mathrm{RT}_{b}$ and on $\mathrm{PC}_{p}$ versus $\mathrm{PC}_{b}$, and these ANOVAs included the repeated-measures factors of task probability (i.e., High-Primary versus High-Background) and response task (i.e., primary versus background), plus a between-subjects factor reflecting the testing order for the two task probabilities (i.e., High-Primary blocks then HighBackground, or the reverse). For these ANOVAs, RTs and PCs were averaged over backward compatibility because it was only defined for the primary task, and they were averaged over SOA and numerical distance because these factors have inherently different effects on the two tasks (i.e., much larger effects on the background task). A second set of ANOVAs considered the primary and background tasks separately (i.e., separate ANOVAs for $\mathrm{RT}_{p}, \mathrm{RT}_{b}, \mathrm{PC}_{p}$, and $\mathrm{PC}_{b}$ ). For these ANOVAs, SOA, numerical distance, and task probability were included as repeated-measures factors, and task probability order was included as a between-subjects factor. An additional factor of backward compatibility was also included in the analysis of primary-task results. Backward compatibility was defined by whether the background-task number was associated with the response key required for the primary task (i.e., compatible), or not (i.e., incompatible). To improve the readability of the results section, we present only the $p$ and $\eta_{p}{ }^{2}$ values of the significant results, all of which are based on $F$ values with degrees of freedom one and $N-2$ (i.e., 60). More detailed ANOVA results are given in the supplemental online materials. 


\section{Results and discussion}

Approximately $0.9 \%$ of correct trials with RTs exceeding $2 \mathrm{~s}$ and $0.07 \%$ of trials with RTs less than $200 \mathrm{~ms}$ were excluded as outliers based on a visual inspection of the obtained RT distributions.

Between-task comparison of RTs Figure 2a shows the overall mean RTs for the primary and background tasks as a function of task probability. To facilitate comparisons across experiments, Fig. 2b-d show the analogous results for Experiments 2-4.

In an ANOVA comparing the means shown in Fig. 2a, with the repeated-measures factors of task probability and response task and the between-subjects factor of the order of testing the two task probabilities, mean $\mathrm{RT}_{p}$ was 39 ms less than mean $\mathrm{RT}_{b}\left(p=.001, \eta_{p}^{2}=0.176\right)$, and responses were $55 \mathrm{~ms}$ faster in the High-Primary blocks than in the High-Background blocks $\left(p<.001, \eta_{p}{ }^{2}=0.419\right)$. Furthermore, the strong crossover interaction shown in the figure was highly significant $\left(p<.001, \eta_{p}^{2}=0.884\right)$, suggesting that the manipulation of task probability effectively modulated the attentional priority given to each task. Clearly, participants tended to respond faster to whichever task was more likely to require a response within both the High-Primary and High-Background conditions. In fact, a specific comparison including only the HighBackground blocks even showed that background-task responses were significantly faster than primary-task responses


Fig. 2 Mean reaction time (RT) in Experiments 1-4 as a function of task probability (High-Primary [HiPri] versus High-Background [HiBac]) and response task (primary $\mathrm{RT}_{p}$ versus background $\mathrm{RT}_{b}$ ). For each in those blocks $\left(p<.001, \eta_{p}^{2}=0.572\right)$. This effect is somewhat surprising given that correct task performance required the primary-task decision to be made before responding to the background task.

The ANOVA comparing the means shown in Fig. 2a also yielded several significant interactions involving the betweensubjects factor of task probability order. Similar interactions were also present in many other ANOVAs reported in this article, for both RTs and PCs, and these interactions reflected only modulations of effect sizes - not qualitative changes in patterns. For that reason, the interactions are of little theoretical interest for present purposes, and they are therefore only reported in the supplemental online materials. As is discussed in more detail in those materials, the interactions involving order could generally be explained either in terms of practice effects (i.e., a tendency for participants to respond faster at later points in the experiment) or in terms of an attentional bias toward whichever task was experienced first as the high-probability task (e.g., a tendency to pay relatively more attention to the primary task throughout the experiment if the High-Primary blocks were tested first than if the HighBackground blocks were tested first, and vice versa).

$\mathbf{R T}_{p}$ Figure 3a shows mean $\mathrm{RT}_{p}$ as a function of task probability and backward compatibility. As was discussed in the introduction, a larger BCE would be expected in the HighBackground blocks if the responses are selected in a more


experiment, the error bar shows two standard errors of the mean based on the pooled error terms for the task probability effect, response task effect, and their interaction 



Fig. 3 Mean reaction time for the primary task $\left(\mathrm{RT}_{p}\right)$ in Experiments 1-4 as a function of task probability (High-Primary [HiPri] versus HighBackground [HiBac]) and backward compatibility (compatible versus

incompatible). For each experiment, the error bar shows two standard errors of the mean based on the pooled error terms for the task probability effect, compatibility effect, and their interaction

parallel fashion in these blocks, with more processing capacity allocated to the numerical judgment task in the HighBackground blocks. To check the statistical reliability of this interaction, a separate ANOVA was carried out on primarytask $\mathrm{RT}_{p}$, with the repeated-measures factors of task probability, SOA, backward compatibility, and numerical distance. This ANOVA yielded a significant $217 \mathrm{~ms}$ effect of task probability $\left(p<.001, \eta_{p}{ }^{2}=0.846\right)$, and primary-task responses were also $26 \mathrm{~ms}$ faster in backward-compatible trials than in backward-incompatible trials $\left(p<.001, \eta_{p}{ }^{2}=0.261\right)$. More importantly, the BCE was reliably larger in HighBackground blocks than in High-Primary blocks $(p=.001$, $\eta_{p}{ }^{2}=0.158$ ), suggesting a greater degree of parallel processing in the former blocks. In addition, this ANOVA produced one further significant interaction involving the BCE. As is shown in Fig. 4, the BCE was larger when numerical distance was far than when it was near $\left(p=.029, \eta_{p}^{2}=0.077\right)$. This interaction is not surprising, because the background-task response could presumably be determined sooner-and thus have more time to produce a $\mathrm{BCE}$ - when the numerical judgment was easier (far) than when it was harder (near).

$\mathbf{R T}_{b}$ Figure 5a shows mean $\mathrm{RT}_{b}$ as a function of task probability and SOA. As was discussed in the introduction, a smaller effect of SOA would be expected in the High-Background blocks if the responses are selected in a more parallel fashion in these blocks, and this interaction is present in the means,

with SOA effects of $105 \mathrm{~ms}$ and $55 \mathrm{~ms}$ in the High-Primary and High-Background blocks, respectively. To check the statistical reliability of this interaction, an ANOVA on the background-task $\mathrm{RT}_{b}$ was computed, with the repeatedmeasures factors of task probability, SOA, and numerical distance. As can be seen in Fig. 5a, background-task responses were $109 \mathrm{~ms}$ faster in the High-Background blocks than in High-Primary blocks $\left(p<.001, \eta_{p}{ }^{2}=0.648\right)$, and they were faster at the $200 \mathrm{~ms}$ SOA than at the $50 \mathrm{~ms} \operatorname{SOA}\left(p<.001, \eta_{p}{ }^{2}\right.$



Fig. 4 Experiment 1 mean reaction time for the primary task $\left(\mathrm{RT}_{p}\right)$ as a function of backward compatibility (compatible versus incompatible) and far versus near numerical distance. The error bar shows two standard errors of the mean based on the pooled error terms for the compatibility effect, the numerical distance effect, and their interaction 



Fig. 5 Mean reaction time for the background task $\left(\mathrm{RT}_{b}\right)$ in Experiments $1-4$ as a function of task probability (High-Primary [HiPri] versus HighBackground $[\mathrm{HiBac}]$ ) and stimulus-onset asynchrony (SOA). For each

experiment, the error bar shows two standard errors of the mean based on the pooled error terms for the task probability effect, SOA, and their interaction

$=0.804)$. More critically, the interaction of task probability and SOA shown in the figure was also significant $(p<.001$, $\eta_{p}{ }^{2}=0.267$ ), providing a further indication that processing was more parallel in the High-Background blocks. This ANOVA also indicated that background-task responses were faster when the numerical distance was far $(M=683 \mathrm{~ms})$ than when it was near $\left(M=774 \mathrm{~ms}, p<.001, \eta_{p}^{2}=0.793\right)$, replicating previous results in numerical discrimination tasks (e.g., Fischer et al., 2007; Hinrichs et al., 1981).

PC results In general, the results obtained in the parallel ANOVAs on PCs were consistent with the RT results with respect to the question of which conditions were easier and which were harder, giving no evidence of speed-accuracy trade-offs. In particular, as was the case for all of the analyses reported in this article, responses were generally less accurate in the slower conditions, as is indicated by strong negative correlations across conditions between RT and PC.

Table 2 summarizes the overall response accuracy for each task and probability condition. In an ANOVA comparing these means, accuracy was higher for primary-task trials $(M$ $=94.0 \%)$ than for background-task trials $(M=92.9 \%, p=$ $.033, \eta_{p}{ }^{2}=0.073$ ), and the crossover interaction between task probability and response task shown in the table was highly

Table 2 Percentage of correct responses (PC) \pm one standard error in Experiments $1-4$ as a function of task probability (High-Primary versus High-Background) and response task (Primary $\mathrm{PC}_{p}$ versus Background $\mathrm{PC}_{b}$ )

\begin{tabular}{lcc}
\hline Task probability & \multicolumn{2}{c}{ Response task } \\
\cline { 2 - 3 } & Primary $\mathrm{PC}_{p}$ & ${\text { Background } \mathrm{PC}_{b}}$ \\
\hline Experiment 1 & & \\
High-Primary & $97.2 \pm 0.28$ & $90.2 \pm 0.86$ \\
High-Background & $90.9 \pm 0.90$ & $95.6 \pm 0.33$ \\
Difference & $6.2 \pm 0.81$ & $-5.4 \pm 0.71$ \\
Experiment 2 & & \\
High-Primary & $96.3 \pm 0.52$ & $81.4 \pm 1.85$ \\
High-Background & $85.6 \pm 1.57$ & $91.7 \pm 0.83$ \\
Difference & $10.6 \pm 1.30$ & $-10.3 \pm 1.42$ \\
Experiment 3 & & $92.7 \pm 1.01$ \\
High-Primary & $95.9 \pm 0.59$ & $94.1 \pm 0.67$ \\
High-Background & $93.6 \pm 0.88$ & $-1.5 \pm 0.71$ \\
Difference & $2.3 \pm 0.53$ & $86.4 \pm 1.01$ \\
Experiment 4 & & $89.4 \pm 0.91$ \\
High-Primary & $94.8 \pm 0.62$ & $-3.0 \pm 0.96$ \\
High-Background & $89.8 \pm 1.09$ & \\
Difference & $4.9 \pm 0.79$ & \\
\hline
\end{tabular}


significant $\left(p<.001, \eta_{p}{ }^{2}=0.605\right)$. Specific comparisons indicated that $\mathrm{PC}_{p}$ was reliably higher than $\mathrm{PC}_{b}$ in High-Primary blocks $\left(p<.001, \eta_{p}{ }^{2}=0.581\right)$, whereas the reverse was true in High-Background blocks $\left(p<.001, \eta_{p}{ }^{2}=0.337\right)$.

$\mathrm{PC}_{p}$ Figure 6a shows primary-task accuracy, $\mathrm{PC}_{p}$, as a function of task probability and backward compatibility. Consistent with the $\mathrm{RT}_{p}$ results, the $\mathrm{BCE}$ on $\mathrm{PC}_{p}$ was larger when there was a higher probability of a background-task response (i.e., larger in High-Background blocks than High-Primary blocks), and this interaction was significant in an ANOVA - parallel to that on $\mathrm{RT}_{p}$ - with repeated-measures factors of task probability, SOA, backward compatibility, and numerical distance $\left(p<.001, \eta_{p}{ }^{2}=0.462\right)$. The BCE was also larger at the short SOA than the long one $\left(p<.001, \eta_{p}{ }^{2}=0.188\right)$. As is often the case with interactions involving PC, however, the interpretations are complicated by the possibility that ceiling effects artificially reduced the differential effects of factors in the most accurate conditions (cf. Loftus, 1978).

In addition, as can be seen in Fig. 7, the ANOVA on $\mathrm{PC}_{p}$ indicated that primary-task responses were more accurate in the High-Primary blocks $(M=97.4 \%)$ than in the HighBackground blocks $\left(M=91.2 \%, p<.001, \eta_{p}{ }^{2}=0.496\right)$, more accurate at the long SOA $(M=95.2 \%)$ than at the short SOA $\left(M=92.9 \%, p<.001, \eta_{p}{ }^{2}=0.223\right)$, and more accurate when the response to that trial's two-digit number was backward-


Fig. 6 Mean percentage correct for the primary task $\left(\mathrm{PC}_{p}\right)$ in Experiments $1-4$ as a function of task probability (High-Primary [HiPri] versus High-Background [HiBac]) and backward compatibility (compatible versus incompatible). For each experiment, the error bar compatible $(M=97.1 \%)$ rather than incompatible $(M=$ $\left.91.0 \%, p<.001, \eta_{p}{ }^{2}=0.553\right)$. The two-way interaction between task probability and SOA was also significant $(p=.011$, $\left.\eta_{p}{ }^{2}=0.103\right)$, with a larger effect of SOA on $\mathrm{PC}_{p}$ in the HighBackground blocks $(3.6 \%)$ than in the High-Primary blocks $(0.8 \%)$. Again, this might be partly due to a ceiling effect, since accuracy approaches $100 \%$ at the longer SOA in the blocks with mostly primary-task responses. The three-way interaction shown in Fig. 7 was also significant $(p=.012$, $\left.\eta_{p}{ }^{2}=0.100\right)$, reflecting the fact that the two-way interaction of compatibility and SOA was larger in the High-Background blocks than in the High-Primary blocks.

Finally, the analysis of $\mathrm{PC}_{p}$ also revealed a significant effect of numerical distance $\left(p=.045, \eta_{p}{ }^{2}=0.066\right)$, with more accurate responses when the distance was near $(M=$ $94.6 \%)$ than when it was far $(M=93.5 \%)$. In addition, the BCE was smaller when the background task number was near the boundary (97.0\% versus $92.2 \%$ ) than when it was far from the boundary ( $97.1 \%$ versus $89.9 \%)$, leading to a significant two-way interaction between backward compatibility and numerical distance $\left(p=.045, \eta_{p}{ }^{2}=\right.$ 0.065). This interaction is to be expected, because background task numbers can be categorized more rapidly when they are far from the boundary, and this gives them more time to influence primary-task responses, which tends to increase the BCE.



shows two standard errors of the mean based on the pooled error terms for the effects of task probability, backward compatibility, and their interaction 




Fig. 7 Experiment 1 percentage of correct primary-task responses $\left(\mathrm{PC}_{p}\right)$ as a function of task probability (High-Primary [HiPri] versus HighBackground $[\mathrm{HiBac}])$, stimulus-onset asynchrony (SOA), and backward

$\mathrm{PC}_{b}$ A further ANOVA parallel to that on background-task $\mathrm{RT}_{b}$ was also carried out on $\mathrm{PC}_{b}$, and it showed only two significant effects. First, participants responded to the background task more accurately when background tasks were common (i.e., High-Background; $M=95.6 \%$ ) than when they were rare (High-Primary; $M=90.2 \%, p<.001, \eta_{p}^{2}=0.479$ ). Second, consistent with previous results in numerical magnitude discrimination tasks (e.g., Hinrichs et al., 1981), the accuracy of these responses was higher when the number was far from the small/large boundary $(M=95.3 \%)$ than when it was near $\left(M=90.5 \%, p<.001, \eta_{p}^{2}=0.448\right)$.

Discussion The main purpose of Experiment 1 was to examine whether increasing the relative frequency of responding to the background task of the PP paradigm would lead to increased evidence of parallel response selection, and there is considerable evidence that it did. First, the effect of SOA on $\mathrm{RT}_{b}$ was smaller in the High-Background blocks than in the HighPrimary blocks (see Fig. 5a). According to the RSB model, second-task RTs are decreased at longer SOAs because there is less waiting for the bottleneck process. The fact that this decrease is smaller in the High-Background blocks thus suggests that there is less waiting in these blocks. Since the primary task actually took longer in these blocks than in the High-Primary blocks, bottleneck models suggest that there should have been more waiting, not less. In contrast, parallel models provide a plausible explanation of the reduced SOA effect, since they do not require the background task to wait for completion of the primary task.

Second, the BCE was larger in the High-Background blocks than in the High-Primary blocks (42 ms versus $9 \mathrm{~ms}$; see Fig. 3a). As was mentioned earlier, there is some debate about whether the BCE can be reconciled with the RSB model in the first place, because the effect seems to suggest that background-task response selection influences the primarytask response selection that supposedly precedes it. Nonetheless, in combination with the changes in $\mathrm{RT}_{p}$ and



compatibility. The error bar shows two standard errors of the mean based on the pooled error terms for the effects of all three factors and their twoway and three-way interactions

$\mathrm{RT}_{b}$ observed when background-task responses are more common, the fact that the $\mathrm{BCE}$ increases in this situation suggests that this effect is driven at least partly by capacitydependent background-task processes operating in parallel with primary-task response selection. In terms of capacity models, the explanation of this interaction is that information about the background-task number was retrieved sooner in the High-Background blocks due to the extra capacity allocated to that task. Being available sooner, the number information would have more opportunity to influence the primary task. Thus, within capacity models, the BCE is larger in HighBackground than in High-Primary for essentially the same reason it is larger at $\mathrm{SOA}=50$ than at $\mathrm{SOA}=200$.

The relative effects of task probability on $\mathrm{RT}_{p}$ versus $\mathrm{RT}_{b}$ provide no evidence of parallel processing, however. As was explained in the introduction, the RSB model predicts a much larger effect of task probability on the primary-task $\mathrm{RT}_{p}$ (Eq. 1) than on the background task $\mathrm{RT}_{b}$ (Eq. 2), and the observed effect sizes of $217 \mathrm{~ms}$ versus $109 \mathrm{~ms}$ are consistent with this prediction. ${ }^{2}$

In addition to these anticipated patterns supporting parallel capacity models, we found additional dramatic (and unexpected) evidence of parallelism in the fact that that backgroundtask responses were substantially faster than primary-task responses in the High-Background blocks where most of the trials required background-task responses. The finding of faster background than primary responses is not difficult to

\footnotetext{
${ }^{2}$ Nonetheless, the $109-\mathrm{ms}$ effect on $\mathrm{RT}_{b}$ still seems quite a bit larger than would be expected from Eq. 2. Consider the individual terms in Eq. 2. One might well expect $\Delta_{\mathrm{Cb}}$ - and also $\Delta_{\mathrm{Cp}}$ - to be near zero. The same motor responses were executed for both tasks, so motor response probabilities were not affected by task probability, and there is no reason why motor execution time would be affected either. One would also expect task probability to have approximately the same effect on the response selection time for both background and primary tasks (i.e., $\Delta_{\mathrm{Bb}} \approx \Delta_{\mathrm{Bp}}$ ). In that case, the observed difference in High-Primary versus High-Background $\mathrm{RT}_{b}$ values would be slightly negative (i.e., $-\Delta_{\mathrm{Ap}}$ ) rather than the $109 \mathrm{~ms}$ observed value. Fortunately, subsequent experiments provide clearer evidence on this point.
} 
understand if responses are selected in parallel (capacity models), because the requirement to make more background-task responses in the High-Background blocks could cause participants to shift capacity from the primary to the background task, thereby speeding the latter relative to the former.

Under the RSB model, however, it is a bit puzzling that background-task responses were faster than primary-task responses in the High-Background blocks. If participants always processed the primary task first, decisions about the background task could only be started after primary-task decisions were completed, and they should thus be slower. One possibility is that participants skipped the primary task on some trials, responding entirely on the basis of the highly probable background task. The $\mathrm{PC}_{p}$ data are inconsistent with this possibility, however. If participants simply responded to the background task, they would always make errors in primary-task trials in the incompatible condition, because the background-task stimulus always indicated an incorrect response in these trials. The actual error rate in these trials averaged only $14 \%$, so it is unlikely that participants skipped the primary task often enough to explain the observed speed-up of the background-task responses in the High-Background blocks. Moreover, the idea that participants sometimes skipped the primary task does not explain why mean $\mathrm{RT}_{p}$ was more than $200 \mathrm{~ms}$ greater in the High-Background blocks than in the High-Primary blocks.

Another possibility for explaining the finding of faster background-task responses than primary-task responses is that participants reversed the order of tasks through the bottleneck with this extreme task probability imbalance (i.e., $80 \%$ versus $20 \%$ ), thus giving bottleneck access first to the background task and then to the primary task. Although such an account might be consistent with the RSB model, many aspects of the results suggest that this did not happen. For example, if it had, then the effect of SOA on $\mathrm{RT}_{b}$ should have disappeared, because background-task processing would not have had to wait for the bottleneck. Instead, $\mathrm{RT}_{p}$ should have increased millisecond for millisecond with SOA, because primary-task processing would have had to wait for the completion of background-task processing. The results are not consistent with either of these predictions. Further evidence about the possibility of task reversal in some or all High-Background trials is discussed in the Appendix.

A final way to explain the High-Background blocks' faster responses to the background task than to the primary task within the RSB model is to assume that in these blocks, primary-task response selection was much faster in no-go trials than in go trials. This is somewhat plausible, because the no-go color appeared in $80 \%$ of trials in these blocks, and participants may have been able to bypass the usual timeconsuming primary-task response selection process when this frequent color appeared. If primary-task response selection was much faster in no-go trials than in go trials, then it would be possible for background task responses - which followed the selection of the primary task no-go responses - to be faster than primary-task responses - which followed the selection of primary-task go responses. Experiment 2 was designed to check further on this account of the fast background-task responses.

In summary, the results of the task probability manipulation support the claim that primary and background tasks can be processed in parallel when the high probability of a background-task response makes it efficient to do so. The key findings in High-Background blocks were that the SOA effect decreased, the BCE increased, and background-task responses were faster than primary-task responses.

\section{Experiment 2}

One of the most striking results of Experiment 1 was that background-task responses were faster than primary-task responses in the High-Background blocks, even though accurate dual-task performance required processing the primary task first. As was already mentioned, one possible explanation for this finding is that participants were able to bypass colortask response selection when they saw the high probability $(80 \%)$ no-go color in the High-Background blocks. Bypassing response selection in these trials could substantially reduce $\mathrm{RT}_{b}$ in comparison with $\mathrm{RT}_{p}$, where response selection would still be needed to choose the left-hand or right-hand response.

As will be reviewed in the General Discussion, many previous results suggest that no-go responses are selected in much the same way as overt responses, making it implausible that primary-task response selection could be bypassed in the background-task trials of Experiment 1. Nonetheless, to examine the plausibility of this explanation of the fast background-task responses, the order of the color and number tasks was reversed in Experiment 2-that is, the number task was the primary task, and the color task was the background task. With this task order, many different numbers were assigned to the primary-task no-go response, and this makes it even less plausible that participants could bypass primarytask response selection, because that option is not signaled by a unique primary-task stimulus. Furthermore, any inherent differences in task difficulty contributing to the reversal of $\mathrm{RT}_{p}$ and $\mathrm{RT}_{b}$ in Experiment 1 should work in the opposite direction in this experiment and eliminate the reversal. Thus, finding a reversal again would support the idea that it was caused by unequal task probabilities rather than a special bypass strategy or unequal task difficulties. Reversing the task order required some changes in the tasks themselves and provided further interesting experimental comparisons, as is described next. 
Background color task Only two colors were needed for the background task - one each assigned to the left-hand and right-hand responses, with no color needed for a no-go response, because there were never no-go stimuli in the background task. Thus, two of the three colors used previously were randomly chosen for each participant and assigned to the left-hand and right-hand responses. Note that in this experiment the BCE corresponds to effects of the left-hand versus right-hand color assignment on numerical judgment task responses. If colors activate their associated responses more rapidly than numbers do, this would increase the BCE relative to Experiment 1.

Primary numerical judgment task More extensive changes were needed to use numerical magnitude as the primary task. Specifically, it was necessary to define three ranges of numbers - one each associated with primary-task left-hand and right-hand responses, and a third range associated with primary-task no-go responses. To make the left-hand and right-hand go-response number ranges symmetric around the no-go range, participants were instructed to respond with the left hand if the number was 34 or less, to respond with the right hand if the number was 75 or more, but not to respond to numbers between 35 and 74 . Thus, intermediate-range numbers were assigned to the primary-task no-go response, and participants were instructed to respond based on the background-task square color if the number fell in this range. Table 3 illustrates the full set of possible conditions.

With numerical discrimination as the primary task, it is possible to compare primary-task responses to numbers near to or far from the decision boundaries of 34 and 75. For example, as can be seen by examining the different conditions listed in Table 3, the primary-task number could be near to a response boundary (e.g., 34) or far from a boundary (e.g., 15), and numerical discriminations should again be more difficult for numbers nearer the boundary. Thus, in this experiment it is possible to assess the effects of numerical distance on primary-task performance.

Interestingly, this experiment also provided a comparison involving the numerical distance effect on performance measures within the background color task (i.e., $\mathrm{RT}_{b}$ and $\mathrm{PC}_{b}$ ). As is shown in Table 3, primary-task no-go numbers could also be nearer to or farther from the boundary (e.g., 36 versus 54), and background-task responses would be required when such numbers were presented. With no-go numbers, numerical distance would presumably affect the speed and accuracy of deciding that no primary-task response was required. Since backgroundtask responses could not be made until the primary-task no-go decision had been reached, the numerical distance effect on primary-task no-go decision time should propagate through to influence $\mathrm{RT}_{b}$ and possibly $\mathrm{PC}_{b}$. The near/far comparison on background-task performance thus provides a measure of the speed and accuracy of making those no-go decisions, and comparable effects of no-go decision difficulty have previously been observed in both PRP (Van Selst \& Johnston, 1997) and PP (Miller \& Durst, 2015) paradigms. Such measures of the time needed for unobserved responses are interesting in their own right and are also useful in the present experiment as a way of validating that participants indeed processed the primary task before the background task, as instructed.

Finally, with numerical discrimination as the primary task, it is also possible to see whether $\mathrm{RT}_{b}$ and $\mathrm{PC}_{b}$ are affected by the compatibility of the no-go number with the background-task response - a novel pattern that we will call the forward compatibility effect (FCE). Specifically, as is illustrated in Table 3, this involves comparing background-task trials where the nogo number was closer to the range associated with the response needed for the background color stimulus or closer to the range associated with the opposite response. Specifically, relatively small no-go numbers (e.g., 35) would be compatible with lefthand responses, whereas relatively large no-go numbers (e.g., 74) would be compatible with right-hand responses. Based on this association of magnitudes and response hands, for example, one would expect some RT facilitation when a small no-go number was presented together with a background-task color requiring the compatible left-hand response, and some RT inhibition when a small no-go number was presented together with a color requiring the incompatible right-hand response. If background-task responses are affected by such facilitation and inhibition, then they should be faster and more accurate in forward-compatible trials than in forward-incompatible trials. There was no analogous FCE in Experiment 1 because there was only a single no-go primary-task stimulus which had no association with either response.

\section{Method}

Except as noted otherwise, the apparatus, stimuli, procedure, and instructions were the same as those used in Experiment 1.

Based on the larger-than-expected effect sizes and smallerthan-expected error terms found in Experiment 1, we estimated that a sample size of approximately 40 would provide greater than $90 \%$ power for observing interactions of the same size as those shown in Figs. 2a and 5a, with $75 \%$ power to detect interactions like that shown in Fig. 3a, so we used this as the target sample size for this and the subsequent experiments. Thirty-nine new participants were recruited from the same pool tested in the earlier experiments. Three participants were excluded for having overall accuracy rates below $80 \%$, and the remaining 36 participants (ages $17-30$ years, $M=20.4$ years, 24 females) were included in the final analyses. Thirty-two were right-handed, and the mean handedness score was $M=60.8$.

Each participant was tested in 10 blocks of the trial types shown in Table 3, with five blocks in one probability condition followed by five blocks in the other, with the order of 
Table 3 Examples of different trial types in Experiment 2

\begin{tabular}{|c|c|c|c|c|c|}
\hline \multicolumn{2}{|l|}{ Stimuli } & \multirow[b]{2}{*}{$\begin{array}{l}\text { Correct } \\
\text { response }\end{array}$} & \multicolumn{3}{|c|}{ Within-block effects } \\
\hline $\begin{array}{l}\text { Primary } \\
\text { task }\end{array}$ & $\begin{array}{l}\text { Background } \\
\text { task }\end{array}$ & & $\begin{array}{l}\text { Numerical } \\
\text { distance }\end{array}$ & $\begin{array}{l}\text { Backward } \\
\text { compatibility }\end{array}$ & $\begin{array}{l}\text { Forward } \\
\text { compatibility }\end{array}$ \\
\hline \multicolumn{6}{|c|}{ Primary-task responses } \\
\hline $15-24$ & Red & Left & Far & Compatible & n.a. \\
\hline $15-24$ & Blue & Left & Far & Incompatible & n.a. \\
\hline $25-34$ & Red & Left & Near & Compatible & n.a. \\
\hline $25-34$ & Blue & Left & Near & Incompatible & n.a. \\
\hline $75-84$ & Blue & Right & Near & Compatible & n.a. \\
\hline $75-84$ & Red & Right & Near & Incompatible & n.a. \\
\hline $85-94$ & Blue & Right & Far & Compatible & n.a. \\
\hline $85-94$ & Red & Right & Far & Incompatible & n.a. \\
\hline \multicolumn{6}{|c|}{ Background-task responses } \\
\hline $35-44$ & Red & Left & Near & n.a. & Compatible \\
\hline $35-44$ & Blue & Right & Near & n.a. & Incompatible \\
\hline $45-54$ & Red & Left & Far & n.a. & Compatible \\
\hline $45-54$ & Blue & Right & Far & n.a. & Incompatible \\
\hline $55-64$ & Red & Left & Far & n.a. & Incompatible \\
\hline $55-64$ & Blue & Right & Far & n.a. & Compatible \\
\hline $65-74$ & Red & Left & Near & n.a. & Incompatible \\
\hline $65-74$ & Blue & Right & Near & n.a. & Compatible \\
\hline
\end{tabular}

Note. Examples of the different conditions for a participant instructed to respond with the left hand if a number of 34 or less was presented, to respond with the right hand if a number of 75 or more was presented, or to respond according to the square color if a number between 35 and 74 was presented (with red assigned to left and blue assigned to right). Within each of these conditions, half of the trials in each block were tested at a stimulus-onset asynchrony (SOA) of $50 \mathrm{~ms}$ and half at an SOA of $200 \mathrm{~ms}$

these conditions counterbalanced across participants. The first block in each probability condition was a half-length practice block, and the other four blocks consisted of 80 trials each. Each of the trial types requiring a primary-task response was tested eight times per High-Primary block and twice per HighBackground block, and these trial numbers were reversed for the types of trials requiring a background-task response.

As in the previous experiment, overall ANOVAs including the repeated-measures factors of task probability and response task and the between-subjects order factor were first carried out to compare performance in the primary and background tasks. Then, additional ANOVAs were conducted separately for the primary and background tasks including the same factors (i.e., SOA, numerical distance, task probability, backward compatibility, and task probability order) as those in Experiment 1 . The additional factor of forward compatibility was included in the ANOVAs of background-task performance (i.e., $\mathrm{RT}_{b}, \mathrm{PC}_{b}$ ).

\section{Results}

Using the same RT outlier cutoffs as in the previous experiment, approximately $2.3 \%$ and $0.1 \%$ of correct trials were excluded as slow and fast outliers, respectively.
Between-task comparison of RTs Figure $2 b$ shows the overall mean RTs for the primary and background tasks as a function of task probability. In an ANOVA with the repeated-measures factors of task probability and response task, mean $\mathrm{RT}_{p}$ was approximately $85 \mathrm{~ms}$ faster than mean $\mathrm{RT}_{b}\left(p<.001, \eta_{p}{ }^{2}=\right.$ 0.508 ), and the crossover interaction shown in the figure was highly significant $\left(p<.001, \eta_{p}{ }^{2}=0.897\right)$. Considering each probability condition separately, primary-task responses were significantly faster than background-task responses in the High-Primary blocks $\left(p<.001, \eta_{p}^{2}=0.841\right)$, and background-task responses were significantly faster than primary-task responses in the High-Background blocks $\left(p<.001, \eta_{p}^{2}=0.436\right)$. Importantly, in a reversal from Experiment 1 , in this experiment task probability had a larger effect on the background task $(206 \mathrm{~ms})$ than on the primary task (144 ms).

$\mathbf{R T}_{p}$ In a separate ANOVA including only primary-task $\mathrm{RT}_{p}$, with the repeated-measures factors of task probability, SOA, backward compatibility, and numerical distance, there were significant main effects of all four experimental factors (i.e., task probability, numerical distance, SOA, and backward 
compatibility) and two significant interactions. Figure $3 \mathrm{~b}$ shows the effects of task probability $\left(p<.001, \eta_{p}{ }^{2}=0.702\right)$, and backward compatibility $\left(p<.001, \eta_{p}{ }^{2}=0.651\right)$, as well as their interaction $\left(p<.001, \eta_{p}{ }^{2}=0.503\right)$. In addition, responses were slightly faster at the short SOA $(M=814 \mathrm{~ms})$ than at the long $\operatorname{SOA}\left(M=840 \mathrm{~ms}, p<.001, \eta_{p}{ }^{2}=0.365\right)$, and they were faster for numbers far from the boundaries $(M=782 \mathrm{~ms})$ than for numbers near the boundaries $\left(M=872 \mathrm{~ms}, p<.001, \eta_{p}{ }^{2}=\right.$ 0.683). The BCE was also larger at the shorter SOA (104 ms) than at the long one (62 ms, $\left.p=.002, \eta_{p}{ }^{2}=0.241\right)$. There was also a tendency for the BCE to be larger with near (101 ms) than far (63 ms) numerical distance, but this effect was only marginally significant $\left(p=.059, \eta_{p}{ }^{2}=0.101\right)$.

$\mathbf{R T}_{b}$ Figure $5 \mathrm{~b}$ shows the effects of task probability and SOA on background-task $\mathrm{RT}_{b}$. An ANOVA on $\mathrm{RT}_{b}$, with the repeated-measures factors of task probability, SOA, numerical distance, and forward compatibility, showed that responses were faster in High-Background blocks $(M=812 \mathrm{~ms})$ than in High-Primary blocks $\left(M=1,016 \mathrm{~ms}, p<.001, \eta_{p}{ }^{2}=0.684\right)$, and they were faster with the long $\operatorname{SOA}(M=867 \mathrm{~ms})$ than the short SOA $\left(M=961 \mathrm{~ms}, p<.001, \eta_{p}{ }^{2}=0.779\right)^{3}$. There was also a numerical tendency for the effect of SOA to be larger in the High-Primary blocks $(M=114 \mathrm{~ms})$ than in the HighBackground blocks ( $M=75 \mathrm{~ms})$, but this interaction was only marginally significant $\left(p=.065, \eta_{p}{ }^{2}=0.100\right)$. This ANOVA also indicated that responses were faster with the far numerical distance $(M=893 \mathrm{~ms})$ than the near distance $(M=936 \mathrm{~ms}, p=$ $\left..003, \eta_{p}{ }^{2}=0.241\right)$, and with forward-compatible $(M=888 \mathrm{~ms})$ than forward-incompatible no-go numbers $(M=941 \mathrm{~ms}, p=$ $\left..001, \eta_{p}{ }^{2}=0.268\right)$. Interestingly, there was also a strong interaction of numerical distance and forward compatibility $(p<$ $\left..001, \eta_{p}{ }^{2}=0.375\right)$. On average, the forward compatibility effect was only $11 \mathrm{~ms}$ with numbers far from the no-go boundaries, whereas it averaged $96 \mathrm{~ms}$ with numbers near these boundaries. This suggests that activation of left-hand and right-hand decisions or responses increased with the time spent deciding whether to choose the no-go response.

PC results Table 2 summarizes overall response accuracy for each task and probability condition. In an ANOVA on the PCs with the repeated-measures factors of task probability and response task, accuracy was higher for primary-task trials $(M$ $=91.0 \%)$ than for background-task trials $(M=86.6 \%, p<$ $\left..001, \eta_{p}{ }^{2}=0.311\right)$, and there was a crossover interaction of this factor with task probability as is shown in the table $(p<$ $\left..001, \eta_{p}{ }^{2}=0.738\right)$. In separate analyses of the two probability

\footnotetext{
${ }^{3}$ One participant was omitted from this analysis because-despite having an overall accuracy rate above $80 \%$ - she had no correct responses in one of the 16 conditions, and she was also omitted from the corresponding analysis of $\mathrm{PC}_{b}$ to keep the RT/PC analyses parallel.
}

conditions, accuracy was significantly higher for the higherprobability task in each condition (both $F>15, p<.001$ ).

$\mathrm{PC}_{p}$ In an ANOVA on primary-task $\mathrm{PC}_{p}$ parallel to that of $\mathrm{RT}_{p}$, SOA produced a significant main effect, with higher accuracy at the longer SOA $(M=91.5 \%)$ than at the shorter one $\left(M=90.4 \%, p=.043, \eta_{p}{ }^{2}=0.115\right)$. This reflects a small speed-accuracy trade-off, since $\mathrm{RT}_{p}$ was also greater at the longer SOA, but the SOA factor was not involved in any interactions. The factors of task probability, numerical distance, and compatibility, however, produced not only strong main effects but also strong interactions. Primary-task responses were more accurate in High-Primary blocks $(M=96.3 \%)$ than in High-Background blocks $(M=85.6 \%$, $\left.p<.001, \eta_{p}{ }^{2}=0.667\right)$, more accurate at the far numerical distance $(M=93.6 \%)$ than the near one $(M=88.3 \%$, $\left.p<.001, \eta_{p}{ }^{2}=0.641\right)$, and more accurate with backwardcompatible $(M=98.0 \%)$ than backward-incompatible $(M=83.9 \%)$ background-task stimuli $\left(p<.001, \eta_{p}{ }^{2}=\right.$ 0.746). Interestingly, the effect of numerical distance was larger in the High-Background blocks than in the HighPrimary blocks $\left(p=.001, \eta_{p}{ }^{2}=0.293\right)$. As can be seen in Fig. 8a-b, the interaction of numerical distance and task probability was present mainly when background-task responses were incompatible, leading to a significant three-way interaction of distance, task probability, and compatibility $(p=.001$, $\left.\eta_{p}{ }^{2}=0.280\right)$. These significant PC interactions cannot be interpreted as reflecting real changes in sensitivity to numerical distance, however, without strong model-based assumptions about the scaling of sensitivity onto PC (cf. Loftus, 1978). For example, the decrease in incompatible-condition accuracy from $97.1 \%$ to $92.1 \%$ in the High-Primary conditions of Fig. $8 \mathrm{a}-\mathrm{b}$ could reflect the same change in sensitivity as the decrease from $80.6 \%$ to $66.0 \%$ in the corresponding High-Background conditions, because of the negatively accelerated function relating $\mathrm{PC}$ to sensitivity.

Figure $8 \mathrm{a}$ and $\mathrm{b}$ also show that the $\mathrm{BCE}$ was larger when background-task responses were more common (i.e., HighBackground as compared with High-Primary; $p<.001, \eta_{p}{ }^{2}$ $=0.696$ ), reinforcing the pattern found with $\mathrm{RT}_{p}$. In addition, the BCE was larger when the numbers were near the decision boundary than when they were far from it $\left(p<.001, \eta_{p}{ }^{2}=\right.$ 0.529 ), presumably because the longer RTs in the near condition allowed more time for the background-task stimulus to produce a compatibility effect.

$\mathrm{PC}_{b}$ Figure $8 \mathrm{c}$ and $\mathrm{d}$ shows the percentages of correct background-task responses, and an ANOVA showed that there were significant main effects in the expected directions for task probability $\left(p<.001, \eta_{p}{ }^{2}=0.592\right)$, forward compatibility $\left(p<.001, \eta_{p}{ }^{2}=0.579\right)$, and numerical distance $(p<$ 



Fig. 8 Experiment 2 percentages of correct responses in the primary task $\left(\mathrm{PC}_{p}\right)$ and in the background task $\left(\mathrm{PC}_{b}\right)$ as a function of task probability (High-Primary [HiPri] versus High-Background [HiBac]), far versus near numerical distance, and forward or backward compatibility. For both $\mathrm{PC}_{p}$

$\left..001, \eta_{p}^{2}=0.679\right)$. All of the two-way interactions were also significant, with larger effects of both forward compatibility $\left(p<.001, \eta_{p}{ }^{2}=0.369\right)$ and numerical distance $\left(p=.003, \eta_{p}{ }^{2}=\right.$ $0.241)$ in the High-Primary blocks than in the HighBackground blocks. In addition, the effect of forward compatibility was larger for the near distance than for the far distance $\left(p<.001, \eta_{p}^{2}=0.532\right)$. Finally, the three-way interaction shown in Fig. $8 \mathrm{c}-\mathrm{d}$ was significant $\left(p=.001, \eta_{p}{ }^{2}=0.269\right)$, indicating that the two-way interaction of compatibility and task probability was larger for near than far numerical distance.

Discussion This experiment replicated most findings of Experiment 1 that were suggestive of parallel processing, despite reversing the primary versus background roles of the color and number tasks. Specifically, background-task responses were faster than primary-task responses in the HighBackground blocks (see Fig. 2b), the BCE was larger in the High-Background blocks than in the High-Primary blocks (see Fig. 3b), and the effect of SOA on $\mathrm{RT}_{b}$ was numerically smaller in the High-Background blocks than in the HighPrimary blocks (see Fig. 5b), although the latter effect was only marginally reliable statistically $(p=.065)$.

A new sign of parallel processing in this experiment is that there was a larger effect of task probability on the background task than on the primary task (206 ms versus $144 \mathrm{~ms})$. As

and $\mathrm{PC}_{b}$, the error bars show two standard errors of the means based on the pooled error terms for the effects of task probability, numerical distance, and compatibility, as well as and their two-way and three-way interactions

noted in the introduction (Eqs. 1 and 2), serial models predict a larger effect of task probability on the primary task, as was found in Experiment 1. Averaging across experiments to counterbalance the identities of the primary and background task, however, it appears that the effects of task probability on primary-task and background-task RTs are actually quite similar (180 ms versus $157 \mathrm{~ms}$ ), contrary to the serial model predictions. Considering the two experiments together, it also appears that the color task is more sensitive to the task probability manipulation than the number task is, but we have no explanation for this difference between the tasks.

\section{Experiments 3 and 4}

Experiments 1 and 2 used the rather extreme task probabilities of $80 \%$ versus $20 \%$ to produce a large imbalance of responses to the two tasks and thereby strongly encourage the allocation of increased capacity to the background task in the HighBackground blocks. Although this manipulation seems to have been successful, one might wonder whether capacity is really gradually adjustable or whether this manipulation was so extreme as to induce participants to use unusual processing strategies in these blocks. For example, participants might have switched a bottleneck process back and forth between the two tasks, much as a single computer CPU can simulate 
parallel processing by switching rapidly back and forth between multiple processes.

To see whether evidence of parallel processing would disappear with a less extreme task probability imbalance, Experiments 3 and 4 were simply replications of Experiments 1 and 2 with a change to $60 \%$ versus $40 \%$ task probabilities. If capacity can be shared gradually across multiple tasks, then these experiments should also produce evidence of increased parallel processing in High-Background blocks relative to High-Primary blocks - albeit not as much of an increase as with the $80 \% / 20 \%$ task probabilities, which would unfortunately tend to reduce the power to detect crucial interactions with task probability. On the other hand, if multitasking performance is not so adjustable - for example, because it is actually limited by an indivisible bottleneck process - then this weaker imbalance might not be sufficient to induce whatever special strategy was responsible for evidence of parallel processing in the earlier experiments.

\section{Method}

Except as noted otherwise, the apparatus, stimuli, procedure, and instructions for Experiments 3 and 4 were the same as those used in Experiments 1 and 2, respectively, except that Experiment 3 included 10 blocks of trials rather than eight. For Experiment 3, color discrimination was the primary task, and 37 new participants were recruited from the same pool tested in the previous experiments. One participant was excluded because of an accuracy rate below $80 \%$, and the remaining 36 participants (ages 19-32 years, $M=20.1$ years, 28 females, 34 right-handed, mean handedness score $M=65.8$ ) were included in the final analyses. For Experiment 4, numerical decision was the primary task, and 43 new participants were recruited. Three participants were again excluded because of accuracy rates below $80 \%$, and the remaining 40 participants (ages 18-41 years, $M=20.3$ years, 36 females, 35 right-handed, mean handedness score $M=60.1$ ) were included in the final analyses.

The major changes in Experiments 3 and 4 relative to Experiments 1 and 2 were in the numbers of trials per block in each of the conditions shown in Tables 1 and 3, which were altered to produce the desired $60 \%$ versus $40 \%$ task probabilities in the High-Primary and High-Background blocks.

The results were analyzed with the same series of ANOVAs used in the earlier experiments. In addition, two further sets of ANOVAs were conducted to make between-experiment comparisons focusing on the effect of the stronger versus weaker task probability manipulations, separately for the primary and background tasks. These included the same factors as the ANOVAs already reported for Experiments 1 and 2, plus an additional between-subjects factor of experiment (i.e., Experiments 1 versus 3 and Experiments 2 versus 4).

\section{Results}

Using the same RT outlier cutoffs as in the previous experiments, approximately $1.5 \%$ and $0.05 \%$ of correct trials were excluded from Experiment 3 as slow and fast outliers, respectively. The corresponding values were $2.7 \%$ and $0.08 \%$ for Experiment 4.

Between-task comparison of RTs Figure $2 \mathrm{c}$ and $\mathrm{d}$ show the overall mean RTs for the primary and background tasks as a function of task probability. In ANOVAs with the repeatedmeasures factors of task probability and response task, mean $\mathrm{RT}_{p}$ was significantly faster than mean $\mathrm{RT}_{b}$ in both Experiment $3\left(p=.009, \eta_{p}^{2}=0.183\right)$, and Experiment $4(p$ $\left.<.001, \eta_{p}{ }^{2}=0.385\right)$. The interactions shown in the figures were also highly significant for both experiments $(p<.001$, $\eta_{p}{ }^{2}=0.525$, and $p<.001, \eta_{p}{ }^{2}=0.585$ ). Considering each probability condition separately, primary-task responses were significantly faster than background-task responses in the High-Primary blocks of both Experiment $3\left(p<.001, \eta_{p}{ }^{2}=\right.$ $0.451)$, and Experiment $4\left(p<.001, \eta_{p}{ }^{2}=0.557\right)$. Background-task responses were not significantly faster than primary-task responses in the High-Background blocks of Experiment $3(p=.317)$, and they were significantly slower in the High-Background blocks of Experiment $4\left(p=.026, \eta_{p}^{2}\right.$ $=0.123$ ). Overall, then, the interactions shown in Fig. 2c-d are weaker than the ones in Fig. 2a-b, consistent with the expectation that there would be weaker parallelism in Experiments 3 and 4 than in Experiments 1 and 2, and the differences in interaction sizes are supported by between-experiment comparisons presented later.

$\mathbf{R T}_{p}$ Figure $3 \mathrm{c}$ and $\mathrm{d}$ show the joint effects of task probability and backward compatibility on $\mathrm{RT}_{p}$. In separate ANOVAs of primary-task $\mathrm{RT}_{p}$ for each experiment with the repeatedmeasures factors of task probability, SOA, backward compatibility, and numerical distance, there were significant effects of task probability for both Experiment $3\left(p=.007, \eta_{p}{ }^{2}=\right.$ $0.198)$, and Experiment $4\left(p=.001, \eta_{p}^{2}=0.262\right)$, showing that the weaker task probability manipulation was still somewhat effective. There were also significant BCEs in both Experiment $3\left(31 \mathrm{~ms}, p<.001, \eta_{p}{ }^{2}=0.496\right)$ and Experiment 4 (92 ms, $\left.p<.001, \eta_{p}{ }^{2}=0.671\right)$. In Experiment 4 the BCE was significantly larger in High-Background blocks than in High-Primary blocks (113 ms versus $70 \mathrm{~ms}, p$ $\left.=.004, \eta_{p}{ }^{2}=0.197\right)$. In Experiment 3, on average across near and far distance numbers, the change in $\mathrm{BCE}$ was numerically in the same direction ( $36 \mathrm{~ms}$ versus $26 \mathrm{~ms}$ ) but not significantly so $(p=.285)$. There was a significant three-way interaction with numerical distance, however $\left(p=.047, \eta_{p}{ }^{2}=0.111\right)$, and the BCE was much larger in High-Background blocks than in High-Primary blocks for far digits ( 55 versus $25 \mathrm{~ms}$ ), even though it was slightly smaller in the High-Background blocks 
for near digits (17 versus $28 \mathrm{~ms}$ ). In Experiment 4 the interaction of BCE and task probability was larger at the short SOA than at the long one $\left(p=.035, \eta_{p}{ }^{2}=0.112\right)$.

These ANOVAs on $\mathrm{RT}_{p}$ also indicated that the interaction between backward compatibility and SOA (not shown) was significant in both experiments $\left(p=.007, \eta_{p}{ }^{2}=0.196\right.$, and $p=$ $\left..008, \eta_{p}{ }^{2}=0.172\right)$, with larger BCEs at the short SOA (50 ms and $105 \mathrm{~ms}$ ) than at the long one (13 $\mathrm{ms}$ and $79 \mathrm{~ms})$, as is generally found (e.g., Fischer et al., 2007; Hommel, 1998; Janczyk, 2016). In Experiment 4, with the number task as primary, there was also the expected significant effect of numerical distance $\left(p<.001, \eta_{p}{ }^{2}=0.840\right)$. In addition, as in Experiment 2, the BCE was larger with near $(108 \mathrm{~ms})$ than far $(75 \mathrm{~ms})$ numerical distance $\left(p=.010, \eta_{p}{ }^{2}=0.161\right)$, consistent with the idea that backward compatibility has more time to produce an effect when the numerical judgment is harder. In this experiment, responses were also $35 \mathrm{~ms}$ slower at the longer SOA than at the shorter one $\left(p<.001, \eta_{p}{ }^{2}=\right.$ $0.473)$.

$\mathbf{R T}_{b}$ Figure 5c and d show the effects of task probability and SOA on background-task $\mathrm{RT}_{b}$. In an ANOVA on the background-task $\mathrm{RT}_{b}$ of Experiment 3 with the same repeated-measures factors used in the parallel analysis of Experiment 1 (i.e., task probability, SOA, and numerical distance), the expected main effects of all three factors were again highly significant. As can be seen in Fig. 5c, responses were faster in the High-Background blocks than in High-Primary blocks $\left(p=.021, \eta_{p}{ }^{2}=0.147\right)$, and they were faster at the $200 \mathrm{~ms}$ SOA than at the $50 \mathrm{~ms} \operatorname{SOA}\left(p<.001, \eta_{p}{ }^{2}=\right.$ $0.862)$. The interaction of these two factors did not approach significance $\left(p=.380, \eta_{p}{ }^{2}=0.023\right)$, however. Backgroundtask responses were also significantly faster when the numerical distance was far $(M=699 \mathrm{~ms})$ than when it was near $(M=$ $\left.784 \mathrm{~ms}, p<.001, \eta_{p}{ }^{2}=0.763\right)$.

In the ANOVA on the background-task $\mathrm{RT}_{b}$ of Experiment 4 parallel to that of Experiment 2, the effect of SOA was significant as usual $\left(p<.001, \eta_{p}{ }^{2}=0.690\right)$. More importantly, the effect of SOA was once again significantly reduced in the High-Background blocks (51 ms) as compared with the HighPrimary blocks (89 ms, $p=.004, \eta_{p}{ }^{2}=0.195$; Fig. 5d). Responses were also faster for forward-compatible trials than forward-incompatible ones ( $p<.001, \eta_{p}{ }^{2}=0.833$ ), and they were faster with numbers far from the relevant boundary rather than near it $\left(p<.001, \eta_{p}{ }^{2}=0.521\right)$. In addition, the forward compatibility effect was smaller in the far condition (42 ms) than the near one $\left(161 \mathrm{~ms}, p<.001, \eta_{p}{ }^{2}=0.681\right)$, again suggesting that primary-task processing produced less activation when it was relatively easy to determine that a no-go number had been presented (i.e., far condition) than when that was relatively hard to determine (i.e., near condition). Finally, the three-way interaction shown in Fig. 9 was significant ( $p=$ $\left..042, \eta_{p}{ }^{2}=0.104\right)$, with a larger compatibility effect at the shorter SOA in the far condition, but a larger compatibility effect at the longer SOA in the near condition. We have no explanation for this pattern.

PC results Table 2 shows overall PCs as a function of task probability and response task. In separate ANOVAs of these values for each experiment, Experiment 3 yielded a significant interaction between task probability and response task $(p<$ $\left..001, \eta_{p}{ }^{2}=0.341\right)$. For this experiment, specific comparisons indicated that $\mathrm{PC}_{p}$ was reliably higher than $\mathrm{PC}_{b}$ in the HighPrimary blocks $\left(p=.004, \eta_{p}{ }^{2}=0.218\right)$, but the advantage for background-task responses was not significant in the HighBackground blocks $(p=.562)$. In Experiment 4 , accuracy was higher in the primary task than in the background task $\left(p<.001, \eta_{p}{ }^{2}=0.339\right)$, and it was higher in High-Primary blocks than in High-Background blocks $\left(p=.049, \eta_{p}{ }^{2}=\right.$ 0.098). The interaction between these two factors shown in Table 2 was also significant $\left(p<.001, \eta_{p}{ }^{2}=0.441\right)$. The simple main effect of response task was significant for HighPrimary blocks $\left(p<.001, \eta_{p}{ }^{2}=0.602\right)$, but not for HighBackground blocks $\left(p=.745, \eta_{p}{ }^{2}=0.003\right)$.

$\mathrm{PC}_{p}$ In the analyses of primary-task $\mathrm{PC}_{p}$ parallel to the $\mathrm{RT}_{p}$ analyses, primary-task responses were more accurate in the High-Primary blocks than in the High-Background blocks in both Experiment $3\left(p<.001, \eta_{p}{ }^{2}=0.358\right)$ and Experiment 4 $\left(p<.001, \eta_{p}{ }^{2}=0.508\right.$; Table 2$)$. They were also $1 \%-2 \%$ more accurate at the long SOA than at the short one in both Experiment $3\left(p<.001, \eta_{p}{ }^{2}=0.414\right)$ and Experiment $4(p=$ $\left..036, \eta_{p}{ }^{2}=0.111\right)$, with the latter effect again reflecting the small speed-accuracy trade-off that was present in Experiment 2. There were BCEs of $5.3 \%$ in Experiment $3(p$ $\left.<.001, \eta_{p}{ }^{2}=0.519\right)$ and $13.0 \%$ in Experiment $4\left(p<.001, \eta_{p}{ }^{2}\right.$ $=0.694)$. The main effect of numerical distance was also significant in both experiments, with a $1.5 \%$ effect on the color task in Experiment $3\left(p<.001, \eta_{p}{ }^{2}=0.352\right)$ and a $5.0 \%$ effect on the numerical judgment task in Experiment $4\left(p<.001, \eta_{p}{ }^{2}\right.$ $=0.756)$. The numerical distance effect was larger for backward-incompatible trials than for backward-compatible trials in both Experiment $3\left(p<.001, \eta_{p}{ }^{2}=0.337\right)$ and Experiment $4\left(p<.001, \eta_{p}{ }^{2}=0.712\right)$. In Experiment 3, where the numerical judgment task was the background task, the BCE was larger when the number was far from the boundary than when it was near to the boundary. This is to be expected, because faster number categorization with far distance gives the number more opportunity to influence the primary color task and thereby produce a larger BCE. Experiment 4, where the numerical judgment task was the primary task, produced the converse pattern: The BCE was larger when the number was near the boundary than when it is far from the boundary. This is also to be expected, because slower judgments about near numbers provide more time for these judgments to be influenced by background-task compatibility and thereby 




Fig. 9 Experiment 4 mean reaction time for the background task $\left(\mathrm{RT}_{b}\right)$ as a function of numerical distance, forward compatibility, and stimulusonset asynchrony (SOA). The error bar shows two standard errors of

produce a larger BCE. The final result in common across experiments was that the BCEs were larger in the HighBackground blocks $(6.6 \%$ and $17.3 \%)$ than in the HighPrimary blocks $(4.0 \%$ and $8.5 \%)$ for both experiments $(p=$ $.018, \eta_{p}^{2}=0.153$, and $\left.p<.001, \eta_{p}{ }^{2}=0.494\right)$.

The ANOVA on $\mathrm{PC}_{p}$ in Experiment 3 revealed two interactions involving backward compatibility, SOA, and numerical distance that were not present in Experiment 4, and these can be seen in Fig. 10. On average, the BCE was larger at the short SOA than at the long one $\left(p=.002, \eta_{p}{ }^{2}=0.253\right)$. In addition, the three-way interaction shown in Fig. 10 was significant $\left(p=.001, \eta_{p}^{2}=0.291\right)$, with a stronger interaction of compatibility and SOA at the far distance than at the near distance.

The analysis of $\mathrm{PC}_{p}$ in Experiment 4 also produced two significant interactions that were not found in Experiment 3, and these can be seen in Fig. 11. First, the effect of task probability was larger at the near distance $(6.3 \%)$ than at the far distance $\left(3.6 \%, p=.006, \eta_{p}^{2}=0.180\right)$. Second, this twoway interaction was larger in backward-incompatible trials than in backward-compatible trials, producing the significant three-way interaction shown in the figure $\left(p=.004, \eta_{p}^{2}=\right.$



Fig. 10 Experiment 3 percentage of correct primary-task responses $\left(\mathrm{PC}_{p}\right)$ as a function of backward compatibility, stimulus-onset asynchrony (SOA), and numerical distance. The error bar shows two standard errors



the mean based on the pooled error terms for the effects of all three factors and their two-way and three-way interactions

0.203). Note that a three-way interaction of the same form was also present in $\mathrm{PC}_{p}$ for Experiment 2 (see Fig. 8a-b).

$\mathrm{PC}_{b}$ The results of the ANOVAs on background-task $\mathrm{PC}_{b}$ with the same factors as the ANOVAs on $\mathrm{RT}_{b}$ showed numerical distance effects of approximately $4 \%$ in both experiments $\left(p<.001, \eta_{p}^{2}=0.589\right.$, and $\left.p<.001, \eta_{p}{ }^{2}=0.563\right)$, and both experiments showed $1.4 \%-3.0 \%$ more accurate responding in High-Background blocks than in High-Primary blocks ( $p=$ $.035, \eta_{p}{ }^{2}=0.124$, and $\mathrm{p}=.003, \eta_{p}{ }^{2}=0.211$ ). In Experiment 4 , the task probability effect was larger at the long SOA $(4.1 \%)$ than at the short one $\left(1.9 \%, p=.047, \eta_{p}^{2}=0.100\right)$. The ANOVA for this experiment also included the forward compatibility factor, which produced a main effect of $10.2 \%$ in the expected direction $\left(p<.001, \eta_{p}{ }^{2}=0.756\right)$. Moreover, in line with the $\mathrm{RT}_{\mathrm{b}}$ results, this effect was larger when the numerical distance was near (15.5\%) than when it was far $(4.9 \%$, $\left.p<.001, \eta_{p}^{2}=0.695\right)$.

Between-experiment comparisons Two further sets of between-experiment ANOVAs were carried out to check which findings were altered significantly by the weaker



of the mean based on the pooled error terms for the effects of all three factors and their two-way and three-way interactions 




Fig. 11 Experiment 4 percentage of correct responses in the primary task $\left(\mathrm{PC}_{p}\right)$ as a function of task probability (High-Primary [HiPri] versus High-Background [HiBac]), numerical distance, and backward

probability manipulation, with each set including separate ANOVAs for $\mathrm{RT}_{p}, \mathrm{RT}_{b}, \mathrm{PC}_{p}$, and $\mathrm{PC}_{b}$. One set compared the results of Experiments 1 versus 3, and the other compared Experiments 2 versus 4 . These ANOVAs had the same factors as those already reported for each of the individual experiments, plus the additional between-subjects factor of experiment (i.e., strong versus weak task probability imbalance). In reporting these analyses, we focus on changes in the differences between the High-Primary and High-Background conditions, and changes between these conditions in the BCE and the SOA effect.

First, as expected, and as is evident in Fig. 2 and Table 2, the weaker manipulations of task probability tended to produce smaller probability effects and smaller interactions involving probability. In the ANOVAs comparing Experiments 1 and 3, there were significant interactions of probability and experiment for $\mathrm{RT}_{p}\left(p<.001, \eta_{p}^{2}=0.430\right)$, for $\mathrm{RT}_{b}\left(p<.001, \eta_{p}^{2}\right.$ $=0.144)$, for $\mathrm{PC}_{p}\left(p=.001, \eta_{p}{ }^{2}=0.110\right)$, and for $\mathrm{PC}_{b}(p=.001$, $\left.\eta_{p}{ }^{2}=0.119\right)$. For the comparison of Experiments 2 and 4, the corresponding interactions were also significant for $\mathrm{RT}_{p}(p=$ $\left..001, \eta_{p}^{2}=0.141\right)$, for $\mathrm{RT}_{b}\left(p<.001, \eta_{p}^{2}=0.335\right)$, for $\mathrm{PC}_{p}(p<$ $\left..001, \eta_{p}{ }^{2}=0.172\right)$, and for $\mathrm{PC}_{b}\left(p<.001, \eta_{p}{ }^{2}=0.194\right)$. These results show that participants were sensitive to the degree of probability imbalance between the two tasks, not just the ordering of tasks as more versus less probable.

Second, the stronger probability manipulation generally produced a stronger tendency for the BCE to be larger in the High-Background blocks than in the High-Primary blocks, leading to significant three-way interactions of backward compatibility, task probability, and experiment on $\mathrm{PC}_{p}$ in Experiments 1 versus $3\left(p=.001, \eta_{p}{ }^{2}=0.103\right)$, on $\mathrm{RT}_{p}$ in Experiments 2 versus $4\left(p=.013, \eta_{p}{ }^{2}=0.083\right)$, and on $\mathrm{PC}_{p}$ in Experiments 2 versus $4\left(p<.001, \eta_{p}^{2}=0.225\right)$. The same trend was present numerically but was not significant for $\mathrm{RT}_{p}$ in the comparison of Experiments 1 versus 3 ( $p=.115$, $\eta_{p}{ }^{2}=0.026$ ). These trends be seen by comparing Fig. $3 \mathrm{a}-\mathrm{b}$ to Fig. $3 \mathrm{c}-\mathrm{d}$ and comparing Fig. $6 \mathrm{a}-\mathrm{b}$ to Fig. $6 \mathrm{c}-\mathrm{d}$.



compatibility. The error bar shows two standard errors of the mean based on the pooled error terms for the effects of all three factors and their two-way and three-way interactions

Third, the strength of the probability manipulation seemed to have some influence on the task probability by SOA interaction with respect to background-task performance. In the analysis of the $\mathrm{RT}_{b}$ patterns shown in Fig. 5, this interaction was stronger in Experiment 1 than $3\left(p=.007, \eta_{p}{ }^{2}=0.075\right)$, but the change in interaction strength did not approach significance in the comparison of Experiments 2 and $4\left(p=.940, \eta_{p}{ }^{2}=0.000\right)$, nor in either of the corresponding analyses of $\mathrm{PC}_{b}$ (both $p \mathrm{~s}>.15$ ).

Discussion Even with the weaker $60 \% / 40 \%$ manipulation of task probability, overall $\mathrm{RT}_{p}$ and $\mathrm{RT}_{b}$ were again sensitive to task probability in both Experiments 3 and 4, though the effects of task probability were smaller than in Experiments 1 and 2, as expected. This pattern is quite consistent with the idea that participants allocated divisible resources to each task roughly in accordance with task probability, since such an allocation strategy would naturally produce a smaller resource imbalance when the task probabilities were less disparate.

Despite the weaker probability manipulation, Experiments 3 and 4 again produced some clear indications of parallel processing, especially Experiment 4 . In this experiment, the backward compatibility effect on $\mathrm{RT}_{p}$ was again larger when background-task responses were more common (see Fig. 3d), particularly at the short SOA where BCEs tend to be stronger in the first place. In addition, background-task $\mathrm{RT}_{b}$ was less affected by SOA when background-task responses were more frequent (see Fig. 5d).

In Experiment 3, neither the BCE nor the effect of SOA on $\mathrm{RT}_{b}$ changed significantly across task probability blocks, so these aspects of the results provided no support for parallel processing. ${ }^{4}$ Nonetheless, the finding that $\mathrm{RT}_{p}$ and $\mathrm{RT}_{b}$ were

\footnotetext{
${ }^{4}$ With these two indications of parallel processing numerically in the same direction as in Experiment 1, however, these two statistically nonsignificant findings may simply reflect a shortage of statistical power. The finding of the corresponding interactions in same direction for both $\mathrm{PC}_{p}$ and $\mathrm{PC}_{b}$ - the former significantly so - makes it more plausible that the expected effects on $\mathrm{RT}_{b}$ were actually present.
} 
approximately equal in the High-Background blocks suggests that there was some parallel response selection these blocks, since the background-task waiting implied by a strictly serial bottleneck would be expected to make $\mathrm{RT}_{b}$ larger than $\mathrm{RT}_{p}$. The finding of approximately equal task probability effects on $\mathrm{RT}_{p}$ and $\mathrm{RT}_{b}$ (47 ms versus $37 \mathrm{~ms}$; see Fig. $2 \mathrm{c}$ ) is also somewhat problematic for RSB models, since these models predict a much larger effect on $\mathrm{RT}_{p}$ (Eqs. 1 and 2).

\section{General discussion}

The present experiments examined the effects of task probability within the PP paradigm in order to look for evidence that multiple responses would be selected in parallel under circumstances where it would be especially efficient to do so. Specifically, it would be efficient to process in parallel, with the majority of processing capacity allocated to the background task, in blocks where most trials required responses to the background task rather than to the primary task (i.e., High-Background blocks). As is reviewed next, several observed results suggest that response selection was indeed parallel in these blocks, at least to a greater extent than in the High-Primary blocks. These results support the conclusions that two responses can be selected in parallel when it is efficient to do so and that relative task probabilities in the PP paradigm provide a robust manipulation of the efficiency of serial versus parallel processing.

By showing that capacity can be flexibly divided across tasks when it is efficient to do so, the present results add to the growing evidence against strict RSB models. If capacity can be divided across tasks, then it cannot be the case that performance is limited by an unavoidable structural bottleneck prohibiting parallel response selection. Because we used arbitrary tasks, the present findings extend the conclusions of previous studies that have found evidence of parallel response selection in dual-task situations with special task combinations (e.g., Göthe, Oberauer, \& Kliegl, 2016; Halvorson, Ebner, \& Hazeltine, 2013; Hazeltine, Ruthruff, \& Remington, 2006; Levy \& Pashler, 2001; Liepelt, Fischer, Frensch, \& Schubert, 2011; Schumacher et al., 2001). Similarly, because our participants had little practice, the findings extend previous conclusions that extensive practice can lead to parallel processing (e.g., Hazeltine, Teague, \& Ivry, 2002; Maquestiaux, LaguëBeauvais, Ruthruff, \& Bherer, 2008; Ruthruff, Johnston, Van Selst, Whitsell, \& Remington, 2003a; Ruthruff, Van Selst, Johnston, \& Remington, 2006; Schubert \& Strobach, 2018; Strobach, Liepelt, Pashler, Frensch, \& Schubert, 2013; Strobach \& Schubert, 2017).

\section{Evidence for parallel response selection}

One indicator of parallel response selection was the increased effect of backward compatibility on $\mathrm{RT}_{p}$ in the High-
Background blocks (Fig. 3) - an increase that was statistically reliable in Experiments 1, 2, and 4. As noted in the introduction, the BCE in the PRP paradigm has often been regarded as an indication of parallel response selection (e.g., Fischer \& Plessow, 2015; Hommel, 2015; Klapp, Maslovat, \& Jagacinski, 2019), although the effect is in principle compatible with serial response selection if it is caused by automatic processing of the second-task stimulus (Hommel, 1998). The present finding that the $\mathrm{BCE}$ increases in blocks where most trials require background-task responses suggests further that it is sensitive to the amount of attentional capacity allocated to the background tasks, as assumed by capacity models with parallel selection of primary-task and background-task responses. More specifically, allocating more capacity to the background task and less to the primary task, as would be expected in these High-Background blocks, would tend to speed background-task processing and slow primary-task processing, both of which would tend to enhance the BCE. Nonetheless, this increase does not rule out models with serial response selection, since it is conceivable that the amount of attention allocated to the background task also influences the strength of the automatic processes driven by it.

A second indicator of parallel response selection was the decreased effect of SOA on $\mathrm{RT}_{b}$ in the High-Background blocks (see Fig. 5), which was significant in Experiments 1 and 4, with results in the same direction in Experiments $2(p=$ $.065)$ and $3(p=.380)$. It is well known that bottleneck models predict that $\mathrm{RT}_{b}$ should decrease sharply as SOA increases due to decreased waiting for access to the response selection stage, with a slope approaching -1 under some circumstances (e.g., Pashler, 1994; Schwarz \& Ischebeck, 2001). Flattening of this slope is thus another indication of parallel response selection that is somewhat difficult to reconcile with bottleneck models (Mattes et al., 2020; Miller et al., 2009; Yildiz \& Beste, 2015).

A third indicator of parallel response selection, which has not been used previously, involved the relative effects of task probability on overall primary-task versus background-task RTs. As explained in the introduction, serial bottleneck models predict that increasing the probability of background-task responses should have a smaller effect on $\mathrm{RT}_{b}$ than on $\mathrm{RT}_{p}$. With regard to $\mathrm{RT}_{b}$, although increased background-task probability would speed the stages required for background-task processing, it would correspondingly slow the stages required for primary-task processing. The net effect would be a small change in $\mathrm{RT}_{b}$, according to bottleneck models, because background-task responses have to wait on the completion of some primary-task stages. More formally, the effects of task probability predicted by serial bottleneck models are summarized in Eqs. 1 and 2, which make it clear that such models predict much larger effects on the primary task than on the background task, in contrast to capacity models which make no such prediction. 
As shown in Fig. 2, task probability did have large effects on RT in both primary and background tasks, but the effects on the background task seem approximately as large as those on the primary task, and the same is true for $\mathrm{PC}_{p}$ and $\mathrm{PC}_{b}$ (see Table 2). In fact, averaging across all four experiments (thereby counterbalancing the identities of the primary and background tasks) and including only the short SOA conditions where the background task should have had to wait on most trials, the effects of task probability on $\mathrm{RT}_{p}$ and $\mathrm{RT}_{b}$ were $115 \mathrm{~ms}$ and $112 \mathrm{~ms}$, respectively, with corresponding effects of $6.0 \%$ and $5.1 \%$ on $\mathrm{PC}_{p}$ and $\mathrm{PC}_{b}$. Considering only the counterbalanced pair of Experiments 1 and 2, which produced large task probability effects, the effects on $\mathrm{RT}_{p}$ and $\mathrm{RT}_{b}$ were $174 \mathrm{~ms}$ and $154 \mathrm{~ms}$, respectively, with effects of $8.4 \%$ and $7.85 \%$ on $\mathrm{PC}_{p}$ on $\mathrm{PC}_{b}$. Such similar values are inconsistent with plausible values of the $\Delta \mathrm{s}$ in Eqs. 1 and 2, and they therefore provide evidence against serial bottleneck models. Within capacity models, on the other hand, $\mathrm{RT}_{b}$ is not constrained by waiting for a bottleneck response selection process, so the finding of comparable task probability effects on $\mathrm{RT}_{p}$ and $\mathrm{RT}_{b}$ is consistent with these models.

Parallel response selection seems especially strongly supported by the finding that background-task responses can be faster than primary-task responses (Experiments 1 and 2). As is illustrated in the bottleneck model stage diagrams of Fig. $1 \mathrm{c}-\mathrm{d}$, in High-Background blocks the processing for both primary-task and background-task responses starts with processing the primary task until the bottleneck finishes, which takes $\mathrm{A}^{*}{ }_{p}+\mathrm{B}_{p}^{*}$ ms. To generate a primary-task response from there requires only an additional $\mathrm{C}_{p}^{*} \mathrm{~ms}$, but to generate a background-task response requires an additional $\mathrm{B}_{b}+\mathrm{C}_{b}$ $\mathrm{ms}$, which should be considerably longer since the decision stage is thought to be the most time-consuming stage in tasks with arbitrary stimulus-response mappings (Luce, 1986). Thus, at least for short SOAs, bottleneck models imply that responses should always be faster in the primary task than in the background task - seemingly regardless of task probability. In the High-Background blocks of Experiments 1 and 2, this prediction was clearly violated regardless of which task was primary and which was background (Fig. 2a-b).

The fact that background-task responses were faster than primary-task responses in the High-Background blocks can be reconciled with capacity models, even though responding correctly required making the primary-task decision before the background-task response, because these models allow parallel processing at the central decision stage. For example, if most central capacity were allocated to the background task, selection of the background-task response could finish before selection of the primary-task response. Then, motor preparation of the background-task response could take place while primary-task response selection finished. When the primarytask no-go response was eventually selected, the backgroundtask response would already have been prepared and could be executed immediately, leading to a fast $\mathrm{RT}_{b}$. When a primarytask go response was selected, however, additional time would be needed to prepare the selected primary-task response, leading to a slower $\mathrm{RT}_{p}$. The process of activating the primary-task response would also presumably be strongly influenced by the previously-activated background task response, producing a large BCE. This tentative account is only one possibility, of course, and further research would be needed to firmly establish a capacity-model account for the observed faster $\mathrm{RT}_{b}$ than $\mathrm{RT}_{p}$ in the High-Background blocks.

\section{Relation of PP and PRP paradigms}

Since the PP paradigm differs from the PRP paradigm in requiring only one response per trial, it is reasonable to consider whether the slowing observed in background-task responses really reflects the same kind of dual-task interference observed in the PRP paradigm. From the perspective of bottleneck models, a key question about the PP paradigm is whether a primary task no-go decision actually requires the central response selection stage at all. If a no-go decision can be made without using the response selection bottleneck, then any $\mathrm{R}_{b}$ slowing observed in this paradigm (e.g., at short SOAs) might be fundamentally different than the $\mathrm{R}_{2}$ slowing observed in PRP paradigms. Some theoretical precedent for this idea comes from Donders (1868/1969), who suggested that no response selection process is actually required in go/no-go tasks. ${ }^{5}$ That precedent concerns pure go/no-go tasks, however, in which there is only a choice between executing or withholding a given known response. It is not clear whether the precedent is applicable to the choice/no-go tasks of the PP paradigm, where the choice is between executing one of two possible overt responses or doing nothing.

Considerable empirical evidence suggests that the no-go responses of the PP paradigm would require an active decision process producing central dual-task interference quite similar to that seen in the PRP task. For example, in pure go/no-go tasks, psychophysiological evidence suggests that there is an active choice between responding and not responding, with the latter likely requiring an inhibitory process (e.g., Gemba \& Sasaki, 1989; Pfefferbaum, Ford, Weller, \& Kopell, 1985; Richer, Silverman, \& Beatty, 1983; Smid, Fiedler, \& Heinze, 2000). Similarly, using the flanker task, Wühr and Heuer (2020) found interference from no-go flankers suggesting that "nogo stimuli and go stimuli are processed in qualitatively the same way and different from neutral stimuli" (p. 526). More directly applicable to dual-tasking, several PRP studies have used pure go/no-go Task $1 \mathrm{~s}$ to compare the effects of motor responding versus not responding on $\mathrm{RT}_{2}$. Even after no-go $\mathrm{R}_{1} \mathrm{~s}, \mathrm{RT}_{2}$ increases substantially as SOA is shortened, suggesting that Task 2 processing is delayed as usual by

\footnotetext{
${ }^{5}$ We thank Hal Pashler for reminding us of this precedent.
} 
the necessity to make a no-go decision about Task 1 (e.g., Bertelson \& Tisseyre, 1969; De Jong, 1993; Van Selst \& Johnston, 1997). In addition, Van Selst and Johnston (1997) varied the difficulty of the decision required to select between the go and no-go responses. This decision difficulty affected $\mathrm{RT}_{2}$ approximately equally following go and no-go $\mathrm{R}_{1} \mathrm{~s}$, again suggesting that selection of the Task 1 no-go response delays $R_{2}$ in much the same way as when an overt $R_{1}$ is actually required.

Previous experiments using the PP paradigm provide even stronger evidence that background-task responses suffer dualtask interference similar to that seen in the PRP paradigm, even though these responses necessarily follow no-go primary task decisions because of the nature of the paradigm. Specifically, although there are some quantitative differences between the PP and PRP paradigms (Miller \& Durst, 2015; Mittelstädt \& Miller, 2017), the PP paradigm also produces three key patterns of results from the PRP paradigm that are commonly regarded as strong support for RSB models (e.g., Pashler, 1994). First, $\mathrm{RT}_{b}$ increases greatly when SOA is short, just as $\mathrm{RT}_{2}$ does in the PRP paradigm. According to RSB models, $\mathrm{RT}_{2}$ increases at short SOAs because Task 2 must wait for access to the central bottleneck, and it would seem parsimonious for the same explanation to apply to $\mathrm{RT}_{b}$. Second, when SOA is short, experimental manipulations that increase the time needed for firststimulus perceptual or central processing also increase $\mathrm{RT}_{b}$. This phenomenon, referred to as effect propagation within the PRP paradigm (e.g., Bausenhart, Rolke, Hackley, \& Ulrich, 2006), is also explained by RSB models in terms of delayed Task 2 access to the central bottleneck (e.g., Pashler, 1992). Specifically, when the early stages of Task 1 processing take $\Delta$ ms longer, Task 2 must wait $\Delta$ ms longer for access to the bottleneck, which increases $\mathrm{RT}_{2}$ by $\Delta \mathrm{ms}$. Again, observing the analogous phenomenon in the $\mathrm{RT}_{b} \mathrm{~s}$ of the PP paradigm (e.g., Miller \& Durst, 2015) suggests that a very similar source of dual-task interference is present even though the primary task requires a no-go response. ${ }^{6}$ Third, experimental manipulations that increase the time needed for perceptual processing of the second stimulus tend to have a larger effect at the longer SOA than at the shorter SOA, whereas manipulations that increase the time needed for second-stimulus processing at later stages (e.g., decision, motor response) tend to have the same effect regardless of SOA. This pattern has also been observed in the PRP paradigm, where it underlies the well-known "locus of slack" logic (e.g., Carrier \& Pashler, 1995; McCann \& Johnston, 1992). RSB models explain this pattern by saying that early second-stimulus perceptual processing can be carried out during a so-called slack period while Task 2 is waiting for access to the serial RSB process. When SOA is short, the slack

\footnotetext{
${ }^{6}$ The effects of primary-task numerical distance on $\mathrm{RT}_{b}$ in the present Experiments 2 and 4 provide further examples of effect propagation within the PP paradigm.
}

period is long, so most of the extra processing needed for a perceptually difficult $\mathrm{S}_{2}$ discrimination can be carried out while Task 2 is waiting for response selection bottleneck process, and this extra processing has little effect on $\mathrm{RT}_{b}$ (for further details, see, e.g., Pashler, 1992). Finding the same pattern in the $\mathrm{RT}_{b} \mathrm{~s}$ of the PP paradigm suggests that the Task 1 no-go decision provides a similar slack period during which $\mathrm{S}_{b}$ perceptual processing can take place, again consistent with the view that similar sources of central dual-task interference operate in the PP and PRP paradigms. In sum, despite the fact that they are only observed after primary-task no-go responses, the $\mathrm{RT}_{b} \mathrm{~S}$ observed in the PP paradigm reproduce three key aspects of the dual-task interference observed in $\mathrm{RT}_{2} \mathrm{~S}$ of the PRP paradigm, suggesting that the central processes responsible for dual-task interference are similar in the two paradigms. Together, these findings suggest that the PP paradigm is a reasonable alternative to the PRP paradigm for studying dual-task interference.

It should also be emphasized that the present evidence of parallel response selection was obtained in conjunction with additional patterns of results that are normally found in PRP tasks and that would normally be regarded as support for RSB models. These additional patterns are important because they extend previous evidence that the PP and PRP paradigms are quite similar (e.g., Miller \& Durst, 2015), which implies that the RSB model should apply to both if it is a general explanation of multi-tasking decrements. For one thing, $\mathrm{RT}_{b}$ was strongly affected by SOA whereas $\mathrm{RT}_{p}$ was little affected. As was reviewed in the introduction, this is the pattern that would be expected if the second task had to wait for access to the response selection bottleneck at short SOAs, so it is usually interpreted as support for RSB models. Furthermore, $\mathrm{RT}_{b}$ was clearly affected by primary-task decision difficulty (i.e., the effect of numeric distance) in Experiments 2 and 4. This propagation of first-task decision difficulty onto second-task RTs is also expected if the second task has to wait for bottleneck access and is also interpreted as support for bottleneck models. Finally, Experiments 2 and 4 showed a forward compatibility effect in which $\mathrm{RT}_{b}$ was affected by the magnitudes of the primary-task no-go numbers. That is, background-task responses were faster when the relatively small or large magnitude of the primarytask no-go number was associated with the same response required for the background task (i.e., forward compatible condition) than when it was associated with the opposite response (i.e., forward incompatible condition). This is strong evidence that primary-task no-go responses were actually selected before background-task responses were made, in accordance with the assumptions underlying the stage diagrams shown in Fig. 1.

\section{Comparison with previous attempts to encourage parallel response selection}

The results of the present experiments support the idea that the capacity allocated to response selection in a given task can be 
modulated by the relative likelihood that the task will actually require a response, which would clearly be an efficient strategy. In contrast, several previous efforts to induce parallel response selection in PRP paradigms were unsuccessful, and it seems theoretically important to know what distinguishes paradigms in which parallel processing is observable from those in which it is not. For example, Ruthruff, Pashler, and Hazeltine (2003b) told participants to give the first and second tasks equal priority, in contrast to the usual PRP instructions that emphasize Task 1. Processing still appeared serial, however. Going even further, Levy and Pashler (2008) tested participants in a driving-like PRP task and instructed them to give maximal priority to the second task. Even with maximal emphasis on the second task, its performance still showed interference at short SOAs, consistent with serial response selection.

As discussed previously, various aspects of the PRP paradigm inherently seem to encourage serial response selection (Meyer \& Kieras, 1997a, 1997b; Miller et al., 2009), and these may contribute to the difficulty of eliciting parallel processing in that paradigm. Some of those aspects are shared by the PP paradigm (e.g., sequential stimulus presentation), but the requirement to make two different responses, which applies only in the PRP paradigm, may be quite important. For example, Miller et al. (2009) showed mathematically that serial response selection would very often be more efficient than limited-capacity parallel response selection when the participants' goal is to minimize the total RT across the two tasks. Under these circumstances, the strategy of serial response selection tends to minimize RT, so serial processing might be used because it is more efficient rather than because it is structurally required. Serial response selection would not be efficient in the High-Background blocks of the PP paradigm, however. Since this paradigm requires only one response per trial, the goal of minimizing total RT across tasks does not apply. Instead, the goal would presumably be to minimize the average RT per trial. To attain that goal, it would clearly be advantageous to increase the limited response selection capacity allocated to the background task when most trials required responses to that task, because the frequent reductions in $\mathrm{RT}_{b}$ would outweigh the rare increases in $\mathrm{RT}_{p}$. Along the same lines, Rieger, Mittelstädt, Dignath, and Kiesel (2020) found that second-task performance is affected by the relative rewards of Task 1 versus Task 2 in the PP paradigm but not in the PRP paradigm, which also suggests that the division of capacity between tasks is more flexible in the PP paradigm. Within the PRP paradigm, it does seem possible to manipulate the degree of parallel processing by varying the proportion of trials with short versus long SOAs (e.g., Miller et al., 2009).

Interestingly, the tendency of the PRP paradigm to encourage serial response selection may be an advantage for investigating issues other than the existence of a structural bottleneck. In particular, the locus-of-slack (e.g., Franz, Sebastian,
Hust, \& Norris, 2008; Tomasik, Ruthruff, Allen, \& Lien, 2009) and effect propagation (e.g., Bausenhart et al., 2006; Janczyk et al., 2018) procedures within the PRP paradigm have proven to be very useful for isolating the mental processes affected by specific experimental manipulations. Both of these procedures rely on the assumption of serial response selection inherent in RSB models, but it is not important for either procedure whether processing is serial because of a structural bottleneck or for some other reason. Therefore, by encouraging serial processing, the PRP paradigm may help to create exactly the conditions needed for these procedures to work effectively, even if those conditions are not required by a structural bottleneck.

\section{Repetition effects}

Although we have emphasized the manipulation of task probability as a way to incentivize differential allocation of capacity to the primary and background tasks, it must be noted that changes in probability are inherently confounded with changes in sequential probabilities (cf. Kornblum, 1973). For example, in the High-Primary blocks most primary-task trials were task repetitions (i.e., followed other primary-task trials), whereas in the High-Background blocks the primary-task trials were mostly task alternations. To some extent, then, effects of task probability could be driven partly by changes in the proportions of task repetitions versus alternations in the two task probability conditions ${ }^{7}$. To the extent that the effects of task sequence also reflect differential capacity allocation (e.g., more capacity allocated to the most recently performed task), of course, effects of task sequence are just as problematic for RSB models as those of task probability.

We reanalyzed the data from all four experiments in an attempt to separate the effects of task probability from those of task repetition versus alternation. Specifically, repeatedmeasures ANOVAs were computed, separately for $\mathrm{RT}_{p}$ and $\mathrm{RT}_{b}$, using individual-participant means on trial $n$ with conditions defined including an additional factor to code the response required in the previous trial $n-1$. Because there were necessarily few trials per participant for repeated trials in low probability tasks, we pooled over some factors (e.g., numeric distance) and included the data from all experiments in a single analysis to maximize power.

The ANOVA on $\mathrm{RT}_{p}$ included the factors of task probability, preceding task, backward compatibility, and experiment. In this analysis, controlling for the preceding task, the BCE was again larger in the High-Background blocks than in the High-Primary blocks $(p<.001)$, demonstrating an influence of task probability on the $\mathrm{BCE}$ beyond that attributable to sequential effects. In addition, the BCE was larger following background-task trial $n-1 \mathrm{~s}$ than following primary-task trial

\footnotetext{
${ }^{7}$ We thank an anonymous reviewer for raising this point.
} 
$n-1 \mathrm{~s}(p<.001)$, suggesting that sequential effects also play a role in modulating this effect.

The ANOVA on $\mathrm{RT}_{b}$ included the factors of task probability, preceding task, SOA, and experiment. Controlling for repetitions in this way, the effect of $\mathrm{SOA}$ on $\mathrm{RT}_{b}$ was again smaller in the High-Background blocks than in the HighPrimary blocks $(p<.001)$, so there was a clear influence of task probability on the SOA effect beyond that attributable to sequential effects. Interestingly, the SOA effect was only marginally smaller following background-task trial $n-1$ s than following primary-task trial $n-1 \mathrm{~s}$, suggesting that sequential effects play little role in modulating this effect.

\section{Implications for number processing}

Although the present study was not primarily concerned with number processing, the pattern of numerical distance effects observed in Experiments 2 and 4 provides new evidence about number processing that dovetails nicely with previous findings (for a recent overview, see Cohen \& Quinlan, 2019). Specifically, these results reinforce previous evidence that determining number magnitude is not a capacity-limited process.

For example, using a variant of the simultaneous/ successive paradigm of Shiffrin and Gardner (1972), BlancGoldhammer and Cohen (2014) found that two-digit comparisons could be made just as well simultaneously as


Fig. 12 Mean reaction time in the primary task $\left(\mathrm{RT}_{p}\right)$ and background task $\left(\mathrm{RT}_{b}\right)$ in Experiments 2 and 4 as a function of task probability (HighPrimary [HiPri] versus High-Background [HiBac]) and numerical successively, which suggests that these digit comparisons are effectively unlimited in capacity. In addition, numerical magnitude can influence RT even when it is task-irrelevant, suggesting that magnitude is processed automatically (e.g., Dehaene \& Akhavein, 1995; Henik \& Tzelgov, 1982). Within the PRP paradigm, there are also reports that the effect of numerical distance on the second task is greatly reduced at short SOA, which further suggests that $\mathrm{S}_{2}$ magnitude information can be processed in parallel with the selection of a Task 1 response (e.g., Fischer et al., 2007; Oriet, Tombu, \& Jolicœur, 2005). Despite being automatic, of course, processing of magnitude information would have to be timeconsuming to produce the numerical distance effects that are commonly observed.

Experiments 2 and 4 provide further evidence that numerical magnitude information is processed automatically. Specifically, in both experiments, the effects of numerical distance on both $\mathrm{RT}_{p}$ and $\mathrm{RT}_{b}$ were approximately additive with those of task probability, as is shown in Fig. 12. As already discussed, various results indicate that less capacity was allocated to the primary number task in the HighBackground blocks, so the numerical distance effects should have been larger in these blocks if these effects arose within a capacity-limited process. This is simply one example of the standard capacity model prediction (e.g., Navon \& Gopher, 1980) that the effects of difficulty manipulations (e.g., near/far



distance (near/far). In each panel, the error bar shows two standard errors of the mean based on the pooled error terms for the task probability effect, numerical distance effect, and their interaction 
numerical distance) should increase when a task is performed with lower capacity. Since the numerical distance were not larger in the High-Background blocks, the present results provide further evidence that numerical magnitude discrimination is a capacity-unlimited process.

\section{Conclusion}

Combining the PP paradigm with a manipulation of task probability effectively encourages parallel response selection in blocks of trials where primary-task responses are rarely required. Under such conditions, the results provide new evidence that response selection for two distinct tasks can be carried out in parallel even with unrelated, unpracticed tasks. These results provide further evidence that bottleneck models - though they provide an excellent account of the serial processing that often occurs - should be regarded as one possible processing option rather than as a reflection of an immutable structural limitation responsible for multitasking decrements. Despite limitations in cognitive capacity, it appears that multiple responses can be selected in parallel when a division of resources across tasks would lead to efficient performance.

Author note We thank Ruben Ellinghaus for assistance in programming the number discrimination task and Patricia Haden, Iring Koch, Anthony Lambert, Victor Mittelstädt, Hal Pashler, Eric Ruthruff, Scott Watter, and three anonymous reviewers for helpful comments on previous versions of the article.

Data availability The data from these experiments are available (https://osf.io/87zu9/). The experiments were not preregistered.

Compliance with ethical standards All experiments were approved by the University of Otago ethics committee and were performed in accordance with the ethical standards described in the 1964 Declaration of Helsinki.

Conflict of interests The authors declare that they have no conflicts of interest with respect to the authorship or publication of this article.

\section{Appendix}

\section{Order of processing in Stage B}

This Appendix examines the possibility that RSB models could account for the effects of the task probability manipulation by assuming that participants sometimes reversed the order of primary-task and background-task bottleneck processing in the High-Background blocks. Figure 13a and b show the stage sequences for primary-task and backgroundtask responses, respectively, under the assumption that the bottleneck processes the background task first (i.e., Stage $\mathrm{B}_{b}$ before Stage $\mathrm{B}_{p}$ ). Some participants could conceivably use this processing order in some trials of the High-Background blocks - either as an explicitly chosen strategy or as an implicit adjustment to task demands- because the outcome of Stage $\mathrm{B}_{b}$ would determine the response in most trials. ${ }^{8}$ Bottleneck models' predictions for these blocks would then be a weighted average of the predictions for this processing order and the predictions for the normal $\mathrm{B}_{p}$-first order, with the weights depending on the proportion of trials in which each processing order was used.

With the reversed processing order, one prediction is that $\mathrm{RT}_{p}$ should increase with SOA. As is shown in Fig. 13a, primary-task processing has to wait on the completion of $\mathrm{B}_{b}$, and this waiting would increase at longer SOAs because Stage $\mathrm{B}_{b}$ would start later-relative to the onset of $\mathrm{S}_{p}$-at those SOAs. In fact, if $\mathrm{B}_{b}$ were processed before $\mathrm{B}_{p}$ in every trial, then $\mathrm{RT}_{p}$ would increase as a function of SOA with a +1 slope, as is indicated by the SOA term in the equation for $\mathrm{RT}_{p, H i B a c}$. Figure 14a shows that this prediction was not confirmed, with little or no increase in the effect of SOA on $\mathrm{RT}_{p}$ in the High-Background blocks relative to the High-Primary blocks.

A second and related prediction is that $\mathrm{RT}_{b}$ should be unaffected by SOA. According to the RSB model, the effect of SOA on $\mathrm{RT}_{b}$ arises because Stage $\mathrm{B}_{b}$ has to wait for the bottleneck to finish with Stage $\mathrm{B}_{p}$. If there is no waiting because $\mathrm{B}_{b}$ is processed first, then the SOA effect should disappear. Figure $14 \mathrm{~b}$ shows that this prediction was also disconfirmed. Although the SOA effects on $\mathrm{RT}_{b}$ were smaller in HighBackground blocks than in High-Primary blocks as expected from parallel models, the effects certainly did not disappear in those blocks as predicted by the reversed-order bottleneck model shown in Fig. 14b.

A third prediction from the reversed processing order account is that $\mathrm{RT}_{p}$ should be affected by background-task difficulty. As can be seen in Fig. 13a, any experimental manipulation that increases the duration of stage $\mathrm{A}_{b}$ or $\mathrm{B}_{b}$ will also increase $\mathrm{RT}_{p}$, since primary-task response selection $\left(\mathrm{B}_{p}\right)$ does not start until background-task response selection $\left(\mathrm{B}_{b}\right)$ has finished. This prediction can be checked using the data of Experiments 1 and 3, in which background-task numerical distance clearly affected the finishing time of $\mathrm{B}_{b}$. If primary-task processing followed background-task processing, then this numerical distance effect

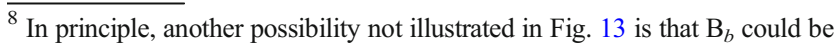
processed first and then the bottleneck might skip Stage $\mathrm{B}_{p}$ completely, allowing stage $C_{b}$ to start immediately after Stage $B_{b}$ was finished. When that happened, participants would simply make whichever response was required for the background task. This might be an efficient processing strategy in the High-Background blocks, because in these blocks the correct response is usually determined by the background task. This possibility will not be considered further, however, because it makes a prediction that is grossly inconsistent with all of our experimental results. Specifically, the prediction is that participants will always make errors in backward-incompatible trials with a primarytask go stimulus (i.e., $0 \%$ correct). In fact, average accuracy rates were always greater than $70 \%$ in these trials, making it clear that participants were rarely, if ever, using this strategy.
} 


$$
\text { a HiBac, } B_{b} \text { before } B_{p}, R_{p}
$$


Fig. 13 Bottleneck model processing sequences leading to responses in the primary $(p)$ and background $(b)$ tasks assuming that there is a structural bottleneck at Stage B, together with the corresponding equations for RTs in the two tasks. The figure illustrates sequences in



Fig. 14 Effects of SOA in the High-Primary (HiPri) and HighBackground (HiBac) blocks of each experiment. a Effects on primarytask $\mathrm{RT}_{p}$. $\mathbf{b}$ Effects on background-task $\mathrm{RT}_{b}$. The error bars show one

should have propagated onto the primary-task $\mathrm{RT}_{p}$ that would have been performed after $\mathrm{B}_{b}$. Relative to this prediction, however, the numerical distance effects on $\mathrm{RT}_{p}$ in Experiments 1 and 3 were $3 \mathrm{~ms}$ in the wrong direction and $14 \mathrm{~ms}$ in the right direction, respectively, in the High-Background blocks. Thus, there was virtually no propagation of the 96-ms and 88-ms numerical distance effects on $\mathrm{RT}_{b}$ found in the same blocks. These results reinforce the conclusion from the SOA effects that participants rarely, if ever, processed the background task before the primary task in the High-Background blocks.

\section{References}

Bausenhart, K. M., Rolke, B., Hackley, S. A., \& Ulrich, R. (2006). The locus of temporal preparation effects: Evidence from the psychological refractory period paradigm. Psychonomic Bulletin \& Review, 13, 536-542. https://doi.org/10.3758/BF03193882

Bertelson, P., \& Tisseyre, F. (1969). Refractory period of C-reactions. Journal of Experimental Psychology, 79(1), 122-128. https://doi. org/10.1037/h0026899

Beste, C., Yildiz, A., Meissner, T. W., \& Wolf, O. T. (2013). Stress improves task processing efficiency in dual-tasks. Behavioural

\section{b HiBac, $B_{b}$ before $B_{p}, R_{b}$}



which Stage $\mathrm{B}_{b}$ is processed before Stage $\mathrm{B}_{p}$, as might be done in the High-Background (HiBac) blocks. a Trials in which a primary-task response is made. $\mathbf{b}$ Trials in which a background-task response is made



standard error of each effect size based on the pooled error terms for the task probability effect, the stimulus-onset asynchrony (SOA) effect, and their interaction, on each measure in each experiment separately

Brain Research, 252, 260-265. https://doi.org/10.1016/j.bbr.2013. 06.013

Blanc-Goldhammer, D. R., \& Cohen, D. J. (2014). Unlimited capacity parallel quantity comparison of multiple integers. Journal of Experimental Psychology: Learning, Memory, \& Cognition, 40(5), 1389-1403. https://doi.org/10.1037/a0036843

Borger, R. (1963). The refractory period and serial choice-reactions. Quarterly Journal of Experimental Psychology, 15, 1-12. https:// doi.org/10.1080/17470216308416546

Brainard, D. H. (1997). The Psychophysics Toolbox. Spatial Vision, 10, 433-437. https://doi.org/10.1163/156856897X00357

Bratzke, D., Rolke, B., \& Ulrich, R. (2009). The source of executionrelated dual-task interference: Motor bottleneck or response monitoring? Journal of Experimental Psychology: Human Perception \& Performance, 35(5), 1413-1426. https://doi.org/10.1037/a0015874

Carrier, L. M., \& Pashler, H. E. (1995). Attentional limits in memory retrieval. Journal of Experimental Psychology: Learning, Memory, \& Cognition, 21, 1339-1348. https://doi.org/10.1037/0278-7393. 21.5.1339

Cohen, D. J., \& Quinlan, P. T. (2019). Limited-capacity identity processing of multiple integers. Attention, Perception, \& Psychophysics, 81(6), 1789-1804. https://doi.org/10.3758/s13414-019-01745-0

De Jong, R. (1993). Multiple bottlenecks in overlapping task performance. Journal of Experimental Psychology: Human Perception \& Performance, 19, 965-980. https://doi.org/10.1037/0096-1523.19.5.965

Dehaene, S., \& Akhavein, R. (1995). Attention, automaticity, and levels of representation in number processing. Journal of Experimental Psychology: Learning, Memory, \& Cognition, 21(2), 314-326. https://doi.org/10.1037/0278-7393.21.2.314 
Donders, F. C. (1969). Over de snelheid van psychische processen [On the speed of mental processes] (W. G. Koster, Trans.). In W. G. Koster (Ed.), Attention and performance II. (pp. 412-431). Amsterdam, Netherlands: North Holland. https://oi.org/10.1016/ 0001-6918(69)90065-1 (Original work published 1868)

Ellenbogen, R., \& Meiran, N. (2008). Working memory involvement in dual-task performance: Evidence from the backward compatibility effect. Memory \& Cognition, 36(5), 968-978. https://doi.org/10. 3758/MC.36.5.968

Fischer, R., Gottschalk, C., \& Dreisbach, G. (2014). Context-sensitive adjustment of cognitive control in dual-task performance. Journal of Experimental Psychology: Learning, Memory, \& Cognition, 40(2), 399-416. https://doi.org/10.1037/a0034310

Fischer, R., \& Hommel, B. (2012). Deep thinking increases task-set shielding and reduces shifting flexibility in dual-task performance. Cognition, 123(2), 303-307. https://doi.org/10.1016/j.cognition. 2011.11.015

Fischer, R., Miller, J. O., \& Schubert, T. (2007). Evidence for parallel semantic memory retrieval in dual tasks. Memory \& Cognition, 35(7), 1685-1699. https://doi.org/10.3758/BF03193502

Fischer, R., \& Plessow, F. (2015). Efficient multitasking: Parallel versus serial processing of multiple tasks. Frontiers in Psychology, 6(1366), 1-11. https://doi.org/10.3389/fpsyg.2015.01366

Franz, E. A., Sebastian, A., Hust, C., \& Norris, T. (2008). Viewer perspective affects central bottleneck requirements in spatial translation tasks. Journal of Experimental Psychology: Human Perception \& Performance, 34, 398-412. https://doi.org/10.1037/0096-1523.34. 2.398

Gemba, H., \& Sasaki, K. (1989). Potential related to no-go reaction of go/ no-go hand movement task with color discrimination in human. Neuroscience Letters, 101(3), 263-268. https://doi.org/10.1016/ 0304-3940(89)90543-0

Giammarco, M., Thomson, S. J., \& Watter, S. (2016). Dual-task backward compatibility effects are episodically mediated. Attention, Perception, \& Psychophysics, 78(2), 520-541. https://doi.org/10. 3758/s13414-015-0998-y

Gleick, J. (1999). Faster: The acceleration of just about everything. New York, NY: Pantheon Books.

Göthe, K., Oberauer, K., \& Kliegl, R. (2016). Eliminating dual-task costs by minimizing crosstalk between tasks: The role of modality and feature pairings. Cognition, 150, 92-108. https://doi.org/10.1016/j. cognition.2016.02.003

Gratton, G., Coles, M. G. H., \& Donchin, E. (1992). Optimizing the use of information: The strategic control of the activation of responses. Journal of Experimental Psychology: General, 121, 480-506. https://doi.org/10.1037/0096-3445.121.4.480

Halvorson, K. M., Ebner, H., \& Hazeltine, E. (2013). Investigating perfect timesharing: The relationship between IM-compatible tasks and dual-task performance. Journal of Experimental Psychology: Human Perception \& Performance, 39(2), 413-432. https://doi. org/10.1037/a0029475

Hazeltine, E., Ruthruff, E. D., \& Remington, R. W. (2006). The role of input and output modality pairings in dual-task performance: Evidence for content-dependent central interference. Cognitive Psychology, 52, 291-345. https://doi.org/10.1016/j.cogpsych.2005. 11.001

Hazeltine, E., Teague, D., \& Ivry, R. B. (2002). Simultaneous dual-task performance reveals parallel response selection after practice. Journal of Experimental Psychology: Human Perception \& Performance, 28, 527-545. https://doi.org/10.1037/0096-1523.28. 3.527

Henik, A., \& Tzelgov, J. (1982). Is three greater than five: The relation between physical and semantic size in comparison tasks. Memory \& Cognition, 10, 389-395. https://doi.org/10.3758/BF03202431

Hinrichs, J. V., Yurko, D. S., \& Hu, J. (1981). Two-digit number comparison: Use of place information. Journal of Experimental
Psychology: Human Perception \& Performance, 7(4), 890-901. https://doi.org/10.1037/0096-1523.7.4.890

Hommel, B. (1998). Automatic stimulus-response translation in dual-task performance. Journal of Experimental Psychology: Human Perception \& Performance, 24, 1368-1384. https://doi.org/10. 1037/0096-1523.24.5.1368

Hommel, B. (2015). Between persistence and flexibility: The yin and yang of action control. In A. J. Elliot (Ed.), Advances in motivation science (Vol. 2, pp. 33-67). New York, NY: Elsevier. https://doi. org/10.1016/bs.adms.2015.04.003

Hommel, B., \& Eglau, B. (2002). Control of stimulus-response translation in dual-task performance. Psychological Research, 66, 260273. https://doi.org/10.1007/s00426-002-0100-y

Hommel, B., Sellaro, R., Fischer, R., Borg, S., \& Colzato, L. S. (2016). High-frequency binaural beats increase cognitive flexibility: Evidence from dual-task crosstalk. Frontiers in Psychology, 7(1287), 1-7. https://doi.org/10.3389/fpsyg.2016.01287

Hübner, R., \& Lehle, C. (2007). Strategies of flanker coprocessing in single and dual tasks. Journal of Experimental Psychology: Human Perception \& Performance, 33(1), 103-123. https://doi. org/10.1037/0096-1523.33.1.103

Janczyk, M. (2013). Who is talking in backward crosstalk? How action goals influence dual-task performance. Presentation at the annual meeting of the Psychonomic Society, Toronto, Canada.

Janczyk, M. (2016). Sequential modulation of backward crosstalk and task-shielding in dual-tasking. Journal of Experimental Psychology: Human Perception \& Performance, 42(5), 631-647. https://doi.org/10.1037/xhp0000170

Janczyk, M., Renas, S., \& Durst, M. (2018). Identifying the locus of compatibility-based backward crosstalk: Evidence from an extended PRP paradigm. Journal of Experimental Psychology: Human Perception \& Performance, 44(2), 261-276. https://doi.org/10. 1037/xhp0000445

Klapp, S. T., Maslovat, D., \& Jagacinski, R. J. (2019). The bottleneck of the psychological refractory period effect involves timing of response initiation rather than response selection. Psychonomic Bulletin \& Review, 26(1), 29-47. https://doi.org/10.3758/s13423018-1498-6

Kleiner, M., Brainard, D. H., Pelli, D. G., Ingling, A., Murray, R., \& Broussard, C. (2007). What's new in psychtoolbox-3? Perception, 36(14), 1. (36 ECVP Abstract Supplement)

Koch, I., Poljac, E., Müller, H., \& Kiesel, A. (2018). Cognitive structure, flexibility, and plasticity in human multitasking-An integrative review of dual-task and task-switching research. Psychological Bulletin, 144(6), 557-583. https://doi.org/10.1037/bul0000144

Kornblum, S. (1973). Sequential effects in choice reaction time: A tutorial review. In S. Kornblum (Ed.), Attention and performance IV (pp. 259-288). New York, NY: Academic Press.

Kunde, W., Wirth, R., \& Janczyk, M. (2018). The role of feedback delay in dual-task performance. Psychological Research, 82, 157-166. https://doi.org/10.1007/s00426-017-0874-6

Lehle, C., \& Hübner, R. (2009). Strategic capacity sharing between two tasks: Evidence from tasks with the same and with different task sets. Psychological Research, 73(5), 707-726. https://doi.org/10. 1007/s00426-008-0162-6

Lehle, C., Steinhauser, M., \& Hübner, R. (2009). Serial or parallel processing in dual tasks: What is more effortful? Psychophysiology, 46(3), 502-509. https://doi.org/10.1111/j.1469-8986.2009.00806.x

Leonhard, T., Ruiz Fernández, S., Ulrich, R., \& Miller, J. O. (2011). Dual-task processing when Task 1 is hard and Task 2 is easy: Reversed central processing order? Journal of Experimental Psychology: Human Perception \& Performance, 37(1), 115-136. https://doi.org/10.1037/a0019238

Levy, J., \& Pashler, H. E. (2001). Is dual-task slowing instruction dependent? Journal of Experimental Psychology: Human Perception \& 
Performance, 27, 862-869. https://doi.org/10.1037/0096-1523.27. 4.862

Levy, J., \& Pashler, H. E. (2008). Task prioritisation in multitasking during driving: Opportunity to abort a concurrent task does not insulate braking responses from dual-task slowing. Applied Cognitive Psychology, 22, 507-525. https://doi.org/10.1002/acp. 1378

Levy, J., Pashler, H. E., \& Boer, E. (2006). Central interference in driving: Is there any stopping the psychological refractory period? Psychological Science, 17(3), 228-235. https://doi.org/10.1111/j. 1467-9280.2006.01690.x

Lieder, F., \& Griffiths, T. L. (2020). Resource-rational analysis: Understanding human cognition as the optimal use of limited computational resources. Behavioral \& Brain Sciences, 43(e1), 1-16. https://doi.org/10.1017/s0140525x1900061x

Liepelt, R., Fischer, R., Frensch, P. A., \& Schubert, T. (2011). Practicerelated reduction of dual-task costs under conditions of a manualpedal response combination. Journal of Cognitive Psychology, 23(1), 29-44. https://doi.org/10.1080/20445911.2011.448025

Loftus, G. R. (1978). On interpretation of interactions. Memory \& Cognition, 6, 312-319. https://doi.org/10.3758/BF03197461

Logan, G. D., \& Burkell, J. (1986). Dependence and independence in responding to double stimulation: A comparison of stop, change, and dual-task paradigms. Journal of Experimental Psychology: Human Perception \& Performance, 12, 549-563. https://doi.org/ 10.1037/0096-1523.12.4.549

Logan, G. D., \& Delheimer, J. A. (2001). Parallel memory retrieval in dual-task situations: II. Episodic memory. Journal of Experimental Psychology: Learning, Memory, \& Cognition, 27, 668-685. https:// doi.org/10.1037/0278-7393.27.3.668

Logan, G. D., \& Gordon, R. D. (2001). Executive control of visual attention in dual-task situations. Psychological Review, 108, 393-434. https://doi.org/10.1037/0033-295X.108.2.393

Logan, G. D., \& Schulkind, M. D. (2000). Parallel memory retrieval in dual-task situations: I. Semantic memory. Journal of Experimental Psychology: Human Perception \& Performance, 26, 1072-1090. https://doi.org/10.1037/0096-1523.26.3.1072

Logan, G. D., \& Zbrodoff, N. J. (1979). When it helps to be misled: Facilitative effects of increasing the frequency of conflicting stimuli in a Stroop-like task. Memory \& Cognition, 7, 166-174. https://doi. org/10.3758/BF03197535

Luce, R. D. (1986). Response times: Their role in inferring elementary mental organization. Oxford, England: Oxford University Press.

Maquestiaux, F., Laguë-Beauvais, M., Ruthruff, E. D., \& Bherer, L. (2008). Bypassing the central bottleneck after single-task practice in the psychological refractory period paradigm: Evidence for task automatization and greedy resource recruitment. Memory \& Cognition, 36(7), 1262-1282. https://doi.org/10.3758/MC.36.7. 1262

Mattes, A., Tavera, F., Ophey, A., Roheger, M., Gaschler, R., \& Haider, H. (2020). Parallel and serial task processing in the PRP paradigm: A drift-diffusion model approach. Psychological Research https:// doi.org/10.1007/s00426-020-01337-w

McCann, R. S., \& Johnston, J. C. (1992). Locus of the single-channel bottleneck in dual-task interference. Journal of Experimental Psychology: Human Perception \& Performance, 18, 471-484. https://doi.org/10.1037/0096-1523.18.2.471

Meyer, D. E., \& Kieras, D. E. (1997a). A computational theory of executive cognitive processes and multiple-task performance: I. Basic mechanisms. Psychological Review, 104, 3-65. https://doi.org/10. 1037/0033-295X.104.1.3

Meyer, D. E., \& Kieras, D. E. (1997b). A computational theory of executive cognitive processes and multiple-task performance: Part 2. Accounts of psychological refractory-period phenomena. Psychological Review, 104, 749-791. https://doi.org/10.1037/ 0033-295X.104.4.749
Miller, J. O. (2006). Backward crosstalk effects in psychological refractory period paradigms: Effects of second-task response types on first-task response latencies. Psychological Research, 70(6), 484 493. https://doi.org/10.1007/s00426-005-0011-9

Miller, J. O. (2017). Psychophysiological measurement of backward response activation in the prioritized processing paradigm. Journal of Experimental Psychology: Human Perception \& Performance, 43(5), 941-953. https://doi.org/10.1037/xhp0000356

Miller, J. O., \& Alderton, M. (2006). Backward response-level crosstalk in the psychological refractory period paradigm. Journal of Experimental Psychology: Human Perception \& Performance, 32(1), 149-165. https://doi.org/10.1037/0096-1523.32.1.149

Miller, J. O., \& Durst, M. (2014). "Just do it when you get a chance": The effects of a background task on primary task performance. Attention, Perception, \& Psychophysics, 76(8), 2560-2574. https://doi.org/10. 3758/s13414-014-0730-3

Miller, J. O., \& Durst, M. (2015). A comparison of the psychological refractory period and prioritized processing paradigms: Can the response-selection bottleneck model explain them both? Journal of Experimental Psychology: Human Perception \& Performance, 41(5), 1420-1441. https://doi.org/10.1037/xhp0000103

Miller, J. O., Ulrich, R., \& Rolke, B. (2009). On the optimality of serial and parallel processing in the psychological refractory period paradigm: Effects of the distribution of stimulus onset asynchronies. Cognitive Psychology, 58(3), 273-310. https://doi.org/10.1016/j. cogpsych.2006.08.003

Mittelstädt, V., \& Miller, J. O. (2017). Separating limits on preparation versus on-line processing in multitasking paradigms: Evidence for resource models. Journal of Experimental Psychology: Human Perception \& Performance, 43(1), 89-102. https://doi.org/10. 1037/xhp0000277

Navon, D. (1984). Resources - A theoretical soup stone? Psychological Review, 91, 216-234. https://doi.org/10.1037/0033-295X.91.2.216

Navon, D., \& Gopher, D. (1980). Task difficulty, resources, and dual-task performance. In R. S. Nickerson (Ed.), Attention and performance VIII (pp. 297-315). Hillsdale, NJ: Erlbaum.

Navon, D., \& Miller, J. O. (2002). Queuing or sharing? A critical evaluation of the single-bottleneck notion. Cognitive Psychology, 44(3), 193-251. https://doi.org/10.1006/cogp.2001.0767

Oldfield, R. C. (1971). The assessment and analysis of handedness: The Edinburgh inventory. Neuropsychologia, 9, 97-113. https://doi.org/ 10.1016/0028-3932(71)90067-4

Oriet, C. J., Tombu, M., \& Jolicœur, P. (2005). Symbolic distance affects two processing loci in the number comparison task. Memory \& Cognition, 33, 913-926. https://doi.org/10.3758/BF03193085

Pashler, H. E. (1992). Attentional limitations in doing two tasks at the same time. Current Directions in Psychological Science, 1, 44-48. https://doi.org/10.1111/1467-8721.ep11509734

Pashler, H. E. (1994). Dual-task interference in simple tasks: Data and theory. Psychological Bulletin, 116, 220-244. https://doi.org/10. 1037/0033-2909.116.2.220

Pashler, H. E., \& Johnston, J. C. (1989). Chronometric evidence for central postponement in temporally overlapping tasks. Quarterly Journal of Experimental Psychology, Section A: Human Experimental Psychology, 41, 19-45. https://doi.org/10.1080/ 14640748908402351

Pelli, D. G. (1997). The Videotoolbox software for visual psychophysics: Transforming numbers into movies. Spatial Vision, 10, 437-442.

Pfefferbaum, A., Ford, J. M., Weller, B. J., \& Kopell, B. S. (1985). ERPs to response production and inhibition. Electroencephalography \& Clinical Neurophysiology, 60, 423-434. https://doi.org/10.1016/ 0013-4694(85)91017-X

Plessow, F., Schade, S., Kirschbaum, C., \& Fischer, R. (2012). Better not to deal with two tasks at the same time when stressed? Acute psychosocial stress reduces task shielding in dual-task performance. 
Cognitive, Affective, \& Behavioral Neuroscience, 12(3), 557-570. https://doi.org/10.3758/s13415-012-0098-6

Reike, D., \& Schwarz, W. (2016). One model fits all: Explaining many aspects of number comparison within a single coherent model-A random walk account. Journal of Experimental Psychology: Learning, Memory, \& Cognition, 42(12), 1957-1971. https://doi. org $/ 10.1037 / \mathrm{xlm} 0000287$

Richer, F., Silverman, C., \& Beatty, J. (1983). Response selection and initiation in speeded reactions: A pupillometric analysis. Journal of Experimental Psychology: Human Perception \& Performance, 9, 360-370. https://doi.org/10.1037/0096-1523.9.3.360

Rieger, T., Mittelstädt, V., Dignath, D., \& Kiesel, A. (2020). Investigating limits of task prioritization in dual-tasking: Evidence from the prioritized processing and the psychological refractory period paradigms. Psychological Research https://doi.org/10.1007/s00426$019-01250-\mathrm{x}$

Rosen, C. (2008). The myth of multitasking. The New Atlantis, 20, 105110.

Ruthruff, E. D., Johnston, J. C., \& Remington, R. W. (2009). How strategic is the central bottleneck: Can it be overcome by trying harder? Journal of Experimental Psychology: Human Perception \& Performance, 35(5), 1368-1384. https://doi.org/10.1037/a0015784

Ruthruff, E. D., Johnston, J. C., Van Selst, M., Whitsell, S., \& Remington, R. (2003a). Vanishing dual-task interference after practice: Has the bottleneck been eliminated or is it merely latent? Journal of Experimental Psychology: Human Perception \& Performance, 29, 280-289. https:// doi.org/10.1037/0096-1523.29.2.280

Ruthruff, E. D., Pashler, H. E., \& Hazeltine, E. (2003b). Dual-task interference with equal task emphasis: Graded capacity sharing or central postponement? Perception \& Psychophysics, 65, 801-816. https:// doi.org/10.3758/BF03194816

Ruthruff, E. D., Pashler, H. E., \& Klaassen, A. (2001). Processing bottlenecks in dual-task performance: Structural limitation or strategic postponement? Psychonomic Bulletin \& Review, 8, 73-80. https:// doi.org/10.3758/BF03196141

Ruthruff, E. D., Van Selst, M., Johnston, J. C., \& Remington, R. (2006). How does practice reduce dual-task interference: Integration, automatization, or just stage-shortening? Psychological Research, 70, 125-142. https://doi.org/10.1007/s00426-004-0192-7

Scherbaum, S., Gottschalk, C., Dshemuchadse, M., \& Fischer, R. (2015). Action dynamics in multitasking: The impact of additional task factors on the execution of the prioritized motor movement. Frontiers in Psychology, 6, 934. https://doi.org/10.3389/fpsyg.2015.00934

Schubert, T., \& Strobach, T. (2018). Practice-related optimization of dual-task performance: Efficient task instantiation during overlapping task processing. Journal of Experimental Psychology: Human Perception \& Performance, 44(12), 1884-1904. https://doi.org/10. 1037/xhp0000576

Schumacher, E. H., Lauber, E. J., Glass, J. M., Zurbriggen, E. L., Gmeindl, L., Kieras, D. E., \& Meyer, D. E. (1999). Concurrent response-selection processes in dual-task performance: Evidence for adaptive executive control of task scheduling. Journal of Experimental Psychology: Human Perception \& Performance, 25, 791-814. https://doi.org/10.1037/0096-1523.25.3.791

Schumacher, E. H., Seymour, T. L., Glass, J. M., Fencsik, D. E., Lauber, E. J., Kieras, D. E., \& Meyer, D. E. (2001). Virtually perfect time sharing in dual-task performance: Uncorking the central cognitive bottleneck. Psychological Science, 12, 101-108. https://doi.org/10. 1111/1467-9280.00318

Schwarz, W., \& Ischebeck, A. (2001). On the interpretation of response time vs onset asynchrony functions: Application to dual-task and precueutilization paradigms. Journal of Mathematical Psychology, 45, 452479. https://doi.org/10.1006/jmps.2000.1336

Shiffrin, R. M., \& Gardner, G. T. (1972). Visual processing capacity and attentional control. Journal of Experimental Psychology, 93, 72-82. https://doi.org/10.1037/h0032453
Sigman, M., \& Dehaene, S. (2005). Parsing a cognitive task: A characterization of the mind's bottleneck. PLOS Biology, 3, 334-349. https://doi.org/10.1371/journal.pbio.0030037

Smid, H. G. O. M., Fiedler, R., \& Heinze, H. J. (2000). An electrophysiological study of the insertion of overt response choice. Journal of Experimental Psychology: Human Perception \& Performance, 26, 1053-1071. https://doi.org/10.1037/0096-1523.26.3.1053

Strobach, T., Liepelt, R., Pashler, H. E., Frensch, P. A., \& Schubert, T. (2013). Effects of extensive dual-task practice on processing stages in simultaneous choice tasks. Attention, Perception, \& Psychophysics, 75(5), 900-920. https://doi.org/10.3758/s13414013-0451-z

Strobach, T., \& Schubert, T. (2017). Mechanisms of practice-related reductions of dual-task interference with simple tasks: Data and theory. Advances in Cognitive Psychology, 13(1), 28-41. https://doi.org/ 10.5709/acp-0204-7

Stürmer, B., Leuthold, H., Soetens, E., Schröter, H., \& Sommer, W. (2002). Control over location-based response activation in the Simon task: Behavioral and electrophysiological evidence. Journal of Experimental Psychology: Human Perception \& Performance, 28, 1345-1363. https://doi.org/10.1037/0096-1523.28.6.1345

Telford, C. W. (1931). The refractory phase of voluntary and associative responses. Journal of Experimental Psychology, 14, 1-36. https:// doi.org/10.1037/h0073262

Thomson, S. J., Danis, L. K., \& Watter, S. (2015). PRP training shows Task 1 response selection is the locus of the backward response compatibility effect. Psychonomic Bulletin \& Review, 22(1), 212218. https://doi.org/10.3758/s13423-014-0660-z

Thomson, S. J., Watter, S., \& Finkelshtein, A. (2010). Parallel response selection in dual-task situations via automatic category-to-response translation. Attention, Perception, \& Psychophysics, 72(7), 17911802. https://doi.org/10.3758/APP.72.7.1791

Tomasik, D., Ruthruff, E. D., Allen, P. A., \& Lien, M. C. (2009). Nonautomatic emotion perception in a dual-task situation. Psychonomic Bulletin \& Review, 16, 282-288. https://doi.org/10. 3758/PBR.16.2.282

Tombu, M., \& Jolicœur, P. (2003). A central capacity sharing model of dual-task performance. Journal of Experimental Psychology: Human Perception \& Performance, 29, 3-18. https://doi.org/10. 1037/0096-1523.29.1.3

Ulrich, R., \& Miller, J. O. (2008). Response grouping in the psychological refractory period (PRP) paradigm: Models and contamination effects. Cognitive Psychology, 57(2), 75-121. https://doi.org/10. 1016/j.cogpsych.2007.06.004

Van Selst, M., \& Johnston, J. C. (1997). Dual-task interference when a response is not required. In M. G. Shafto \& P. Langley (Eds.), Proceedings of the nineteenth annual conference of the cognitive science society (pp. 787-792). Mahwah, NJ: Erlbaum. Retrieved from https:/ntrs.nasa.gov/archive/nasa/casi.ntrs.nasa.gov/20020045203.pdf

Watter, S., \& Logan, G. D. (2006). Parallel response selection in dual-task situations. Perception \& Psychophysics, 68, 254-277. https://doi. org/10.3758/BF03193674

Welford, A. T. (1952). The "psychological refractory period" and the timing of high-speed performance-A review and a theory. British Journal of Psychology, 43, 2-19. https://doi.org/10.1111/j.20448295.1952.tb00322.x

Wickens, C. D., Goh, J., Helleberg, J., Horrey, W., \& Talleur, D. A. (2003). Attentional models of multi-task pilot performance using advanced display technology. Human Factors, 360-380. https:// doi.org/10.4324/9781315092898-10

Wühr, P., \& Heuer, H. (2020). To respond or not to respond? A model-based comparison between the processing of go, nogo, and neutral stimuli. Journal of Experimental Psychology: Human Perception \& Performance, 46(5), 525-549. https:// doi.org/10.1037/xhp0000731 
Yildiz, A., \& Beste, C. (2015). Parallel and serial processing in dualtasking differentially involves mechanisms in the striatum and the lateral prefrontal cortex. Brain Structure and Function, 220(6), 3131-3142. https://doi.org/10.1007/s00429-014-0847-0

Zwosta, K., Hommel, B., Goschke, T., \& Fischer, R. (2013). Mood states determine the degree of task shielding in dual-task performance.
Cognition \& Emotion, 27(6), 1142-1152. https://doi.org/10.1080/ 02699931.2013 .772047

Publisher's note Springer Nature remains neutral with regard to jurisdictional claims in published maps and institutional affiliations. 